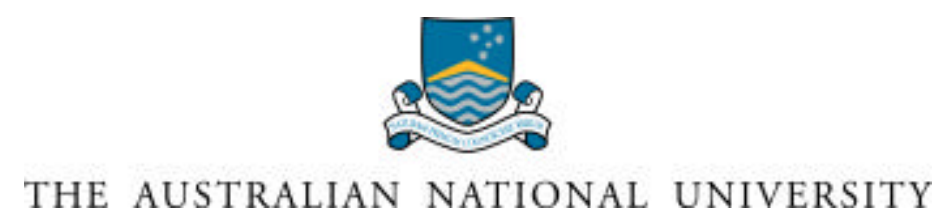

Working Paper Series in Finance 99-06

\title{
A ROBUST ALGORITHM IN SEQUENTIALLY SELECTING SUBSET TIME- SERIES SYSTEMS USING NEURAL NETWORKS
}

\author{
Jack H.W. Penm, T.J. Brailsford and R.D. Terrell
}

Faculty of Economics and Commerce

The Australian National University

JEL Classification

Keywords

Last revision date:

Contact author:
$\mathrm{C} 32, \mathrm{C} 45, \mathrm{G} 10$

Economic and Financial Forecasting; Neural Networks;

Subset VAR and VRDL Modelling

May 1999

Dr Jack Penm

Faculty of Economics and Commerce

The Australian National University

Canberra ACT 0200 AUSTRALIA

Telephone: 0262490535 (International +6126249 0535)

Facsimile: 0262490414 (International +61 26249 0414)

Email: jack.penm@anu.edu.au

The working paper series is for manuscripts in draft form. Comments are welcome and should be sent to the contact author. Please do not quote without permission.

(C) Copyright 1999 Jack H.W. Penm, T.J. Brailsford and R.D. Terrell 


\title{
A ROBUST ALGORITHM IN SEQUENTIALLY SELECTING SUBSET TIME- SERIES SYSTEMS USING NEURAL NETWORKS
}

\author{
Jack H.W. Penm, T.J. Brailsford and R.D. Terrell \\ Faculty of Economics and Commerce \\ The Australian National University
}

\begin{abstract}
In this paper a numerically robust lattice-ladder learning algorithm is presented that sequentially selects the best specification of a subset time series system using neural networks. We have been able to extend the relevance of multi-layered neural networks and so more effectively model a greater array of time series situations. We have recognised that many connections between nodes in layers are unnecessary and can be deleted. So we have introduced inhibitor arcs - reflecting inhibitive synapses. We also allow for connections between nodes in layers which have variable strengths at different points of time by introducing additionally excitatory arcs - reflecting excitatory synapses. The resolving of both time and order updating leads to the optimal synaptic weight updating and allows for the optimal dynamic node creation/deletion within the extended neural network. The paper presents two applications that demonstrate the usefulness of the process.
\end{abstract}




\section{INTRODUCTION}

Neural networks have become increasingly important in recent years in both stationary and non-stationary time series applications such as economic and financial forecasting, computing and signal processing (see Refenes et al (1996)). Neural networks with their modelling and forecasting ability create a need for time series analysts to relate neural networks and time series modelling techniques. Chakraborty et al (1992) have shown that neural networks can be suitable for forecasting multivariate time series. Teräsvirta et al (1993) demonstrated the power of the neural network linearity test. In this paper, we introduce an extension to the structure of neural networks to increase their modelling power for subset time series systems.

Consider a vector time series model of the form

$$
\mathrm{z}(\mathrm{t})+\sum_{\mathrm{i}=1}^{\mathrm{p}} \mathrm{h}_{\mathrm{i}}^{\prime} \mathrm{y}(\mathrm{t}+1-\mathrm{i})=\varepsilon^{\mathrm{h}}(\mathrm{t}),
$$

where $h_{i}^{\prime}, i=1,2, \ldots, p$ are gxr parameter matrices, $\varepsilon^{h}(t)$ is a gx1 stationary process with $\mathrm{E}\left\{\varepsilon^{\mathrm{h}}(\mathrm{t})\right\}=0$ and

$$
\mathrm{E}\left\{\varepsilon^{\mathrm{h}}(\mathrm{t}) \varepsilon^{\prime \mathrm{h}}(\mathrm{t}-\tau)\right\}= \begin{cases}\Omega & \tau=0 \\ 0 & \tau \neq 0\end{cases}
$$

The equation (1.1) and properties associated with $\varepsilon^{\mathrm{h}}(\mathrm{t})$ together constitute a vector rational distributed lag model (VRDL), which involves a g-dimensional regressand vector $\mathrm{z}(\mathrm{t})$ and an r-dimensional regressor vector $y(t)$. In practice, the assumption that $E\left\{\varepsilon^{\mathrm{h}}(\mathrm{t})\right\}=0$ means that the vector data of both $z(t)$ and $y(t)$ will have to be mean corrected. For simplicity we ignore mean correction below. It would make no difference to any of the findings presented in this paper. 
In subset VRDL modelling, we consider the subset VRDL system whose predictor is of the form

$$
\hat{\mathrm{z}}(\mathrm{t})=-\sum_{\mathrm{i}=1}^{\mathrm{p}} \mathrm{h}_{\mathrm{i}}^{\prime}\left(\mathrm{I}_{\mathrm{s}}\right) \mathrm{y}(\mathrm{t}+1-\mathrm{i})
$$

where $h_{i}^{\prime}\left(I_{s}\right)=0$ if $i \in I_{s}$. Thus $I_{s}$ specifies the integers between 1 and $p-1$ that correspond to excluded entries. ${ }^{1}$ A VRDL model can serve as an infinite moving average representation of a rational vector AR, ARMA or ARMAX model. The use of VRDL models in economic and financial time series is versatile. Mittnik (1989) successfully applied the VRDL to time series forecasting using balanced state space representations. Holmes and Hutter (1989) suggested the use of a subset VRDL system to assess the relationship between $\mathrm{z}(\mathrm{t})$ and the set of current and lagged $\mathrm{y}(\mathrm{t})$ where there is a continuous or a random delay.

Watanabe et al (1992) studied a two-layered linear neural network as a physical structure of a full-order autoregressive moving-average model and found that no hidden layer suffices in the network for the learning requirements as $y(t)$ comprises the first-order terms $\mathrm{y}_{1}(\mathrm{t}), \mathrm{y}_{2}(\mathrm{t}), \ldots, \mathrm{y}_{\mathrm{r}}(\mathrm{t})$. In this paper, we develop an extended two-layered neural network that can be easily applied to a time series system with a subset structure. To achieve this objective, we introduce two types of connection, namely inhibitor arc and switchable connection, to extend the neural network structure. The inhibitor arc was introduced to network theory by Petri (1979) and the associated connection strength for all these arcs is constrained to zero at all times. The switchable connection is obtained from switching theory and the strength is switchable between zero and non-zero at any time. The introduction of these connections increases the modelling and analysis power of the neural networks.

\footnotetext{
1 We must choose a maximum lag to initialise the lattice-ladder learning algorithm as proposed in section 3 . That is, therefore, a natural way to use the concept of the exclusion set for programming the proposed algorithm.
} 
If $y(t)$ does not only include the first-order terms, but also contains the second-order terms $y_{1}(t) y_{2}(t), y_{1}^{2}(t)$ and $y_{2}^{2}(t)$ and so on, then a polynomial neural network, with a single hidden layer investigated in Kwok and Yeung(1997), can be constructed as a physical structure of the subset VRDL model of (1.1). The hidden-node transfer function consists of a quadratic regression polynomial of two variables, and this network can approximate any nonlinear function to a desired degree of accuracy. Section 2 details this development.

It is often the case that a VRDL model, which works well in explaining the behaviour of a system over a specific sample, may evolve over time because of political, economic, environmental or other external changes. To incorporate such evolution, a learning algorithm is often included in a VRDL model so that the model can be updated over time. There are many well developed computationally efficient and numerically robust recursive algorithms which can be employed to update the VRDL models (see Carayannis et al 1986). However, most of these algorithms are only applicable to full order models. In subset VRDL modelling, the commonly used learning algorithms for full order models are not applicable because the structure of the lag coefficients is estimated without the 'presence and absence' restrictions. As a result, it is necessary to develop a learning algorithm for subset VRDL models which include full-order models as a special case. In section 3, we focus on a double (a-priori and a-posterior) lattice-ladder algorithm. The algorithm does not need to update the angle variable, but carries out in a more direct way the update of the optimal structure. This algorithm, which computes concurrently both the a-priori and at posterior residuals in a recursion cycle, possesses better numerical accuracy and is less sensitive to roundoff errors than direct matrix inversion counterparts. In addition, only the lattice algorithm is required for VAR modelling. 
The remainder of the paper is organised as follows. Section 2 introduces the extended neural network structure for subset time-series modelling. Section 3 demonstrates the overall lattice-ladder learning algorithm for extended neural networks. Section 4 provides an illustration, in which the proposed learning algorithm is used to describe the provision of all possible lattice-ladder structures for subset VRDL modelling and all possible lattice structures for subset VAR modelling. To demonstrate the usefulness of this algorithm, two applications are presented in section 5 .

\section{EXTENDED NEURAL NETS}

Neural nets can be used as a tool for the study of time-series systems. A two-layered neural network proposed by Watanabe et al (1992) provides a typical example which is simple, user-friendly and powerful. However, this net has difficulty in practical modelling of subset time-series systems. In this section, the net is extended to increase its modelling power in this area. Constraints on the connection strength (synaptic weights) are imposed on the network structure. Two types of connection (synapse), namely inhibitor arc and switchable connection, are incorporated into the neural net structure. Figure 1 shows both types of connection in the extended two-layered neural network for a specified illustrative subset VRDL model. 
Figure 1: Extended two-layered neural network.

This linear neural network computes the following 3rd order system:

$$
\hat{\mathrm{z}}(\mathrm{t})=-\mathrm{h}_{1} \mathrm{y}(\mathrm{t})-\mathrm{h}_{3} \mathrm{y}(\mathrm{t}-2),
$$

where $\mathrm{r}=1, \mathrm{~g}=1$ and $\mathrm{h}_{2}=0$. A switchable connection has an arrowhead and an inhibitor arc has a small solid black circle at the output node.

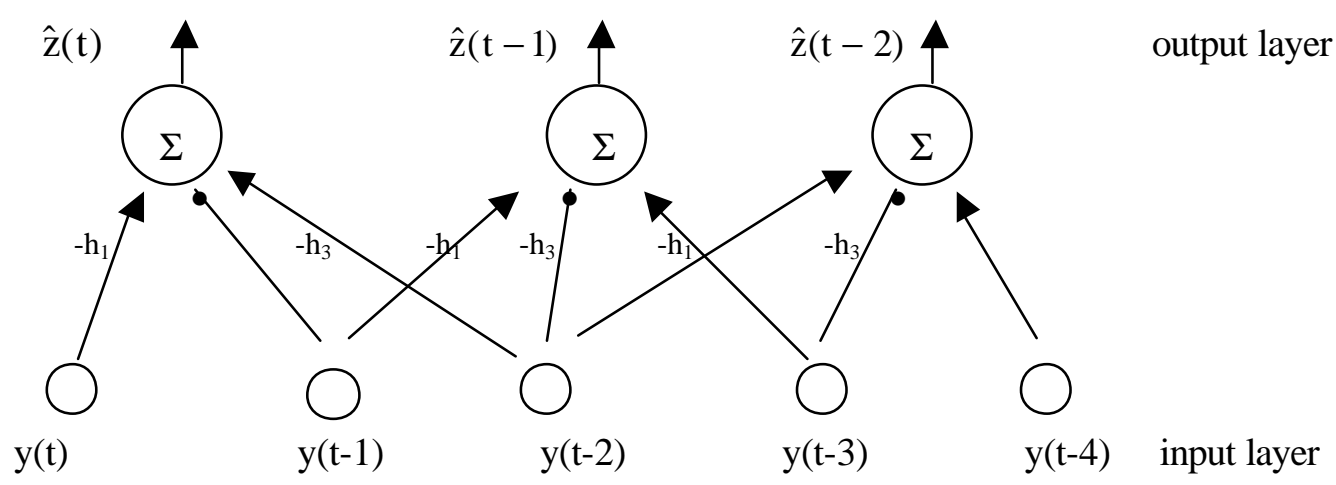

In Figure 1, the higher layer is the output layer, and the lower one is the input layer. All nodes in each layer express artificial neural units. Each unit in the output layer represents a neural output vector, $\hat{\mathrm{z}}(\mathrm{t})$ or its lagged vector. $\hat{\mathrm{z}}(\mathrm{t})$ receives inputs from the input units represented by the current and the second lagged neural input-vectors. A switchable connection from an input node to the output node has an arrowhead at the output node, and the associated connection strength is switchable between zero and non-zero at any time. An inhibitor arc from an input node to the output node has a small solid black circle rather than an arrowhead at the output node. This circle means 'not connected' and the associated connection strength is constrained to zero at all times. Each connection to $\hat{\mathrm{Z}}(\mathrm{t})$ performs a linear transformation determined by the connection strength $h$, so that the total input for the output unit $\hat{\mathrm{z}}(\mathrm{t})$ is $-\mathrm{h}_{1} \mathrm{y}(\mathrm{t})-\mathrm{h}_{2} \mathrm{y}(\mathrm{t}-1) \cdots-\mathrm{h}_{\mathrm{p}} \mathrm{y}(\mathrm{t}+1-\mathrm{p})$. If $\mathrm{h}_{\mathrm{k}}=0$ at $\mathrm{t}=\mathrm{T}_{1}$, the associated connection is inhibitive; if $h_{k} \neq 0$ at $t=T_{1}+d T$, the associated connection is excitatory. It is noteworthy that $\mathrm{dT}$ is sufficiently large enough to ensure that the underlying relationships between $z(t)$ and $y(t)$ change smoothly and gradually. If, however, $h_{k}=0$ at all times, the associated connection becomes an inhibitor arc. 
The extended network has a dynamic setting, that is, the 'presence and absence' restrictions on the coefficients of the optimal VRDL model may update each time a new observation becomes available. This pattern update reflects that some synapses interacting between neurons in the input layer and neurons in the output layer switch between the excitatory and inhibitive states. This specification is superior to the conventional static one in which no 'absence' restrictions are imposed on the coefficients and consequently all synapses are excitatory.

We have demonstrated a subset VRDL model as a basis for constructing an extended twolayered neural network. If the neural input-vector $y(t)$ includes the first-order and secondorder terms $y_{1}(t), y_{2}(t), y_{1}(t) y_{2}(t), y_{1}^{2}(t)$ and $y_{2}^{2}(t)$ and so on, the VRDL model can be used to construct a three-layered polynomial neural network. The hidden-node transfer function in this network consists of a quadratic regression polynomial of two variables used by the group method of data handling (GMDH) algorithm of Ivakhnenko (see Farlow (1984)). General connection between the mean-corrected input and output vectors can be expressed as

$$
\mathrm{f}_{\mathrm{h}}\left(\mathrm{y}_{1}(\mathrm{t}), \mathrm{y}_{2}(\mathrm{t})\right)=h_{11} \mathrm{y}_{1}(\mathrm{t})+h_{12} \mathrm{y}_{2}(\mathrm{t})+h_{13} \mathrm{y}_{1}(\mathrm{t}) \mathrm{y}_{2}(\mathrm{t})+h_{14} \mathrm{y}_{1}^{2}(\mathrm{t})+h_{15} \mathrm{y}_{2}^{2}(\mathrm{t})
$$

where $\mathrm{y}_{\mathrm{i}}(\mathrm{t}), \mathrm{i}=1,2$, are the input variables and $h_{1 \mathrm{i}}, \mathrm{i}=1,2, \ldots, 5$ are coefficients. The proposed construction method is simple to use and can be applied to an M-layered polynomial neural network with hidden layer nodes in layer $m \in[1, \mathrm{M}-2]$. Figure 2 illustrates the structure of a polynomial network with a single hidden-layer for the predictor of a VRDL model

$$
\hat{z}(t)=-h_{1}^{\prime} y(t)-h_{2}^{\prime} y(t-1)-h_{3}^{\prime} y(t-2),
$$

where $y(t)=\left[\begin{array}{lllll}y_{1}(t) & y_{2}(t) & y_{1}(t) y_{2}(t) & y_{1}^{2}(t) & y_{2}^{2}(t)\end{array}\right]^{\prime}$ and $h_{i}^{\prime}=\left[\begin{array}{ll}h_{i, 1} & h_{i, 2} \cdots h_{i, 5}\end{array}\right], i=1,2$ and 3 
Figure 2: A polynomial neural network with a single hidden-layer for a VRDL model

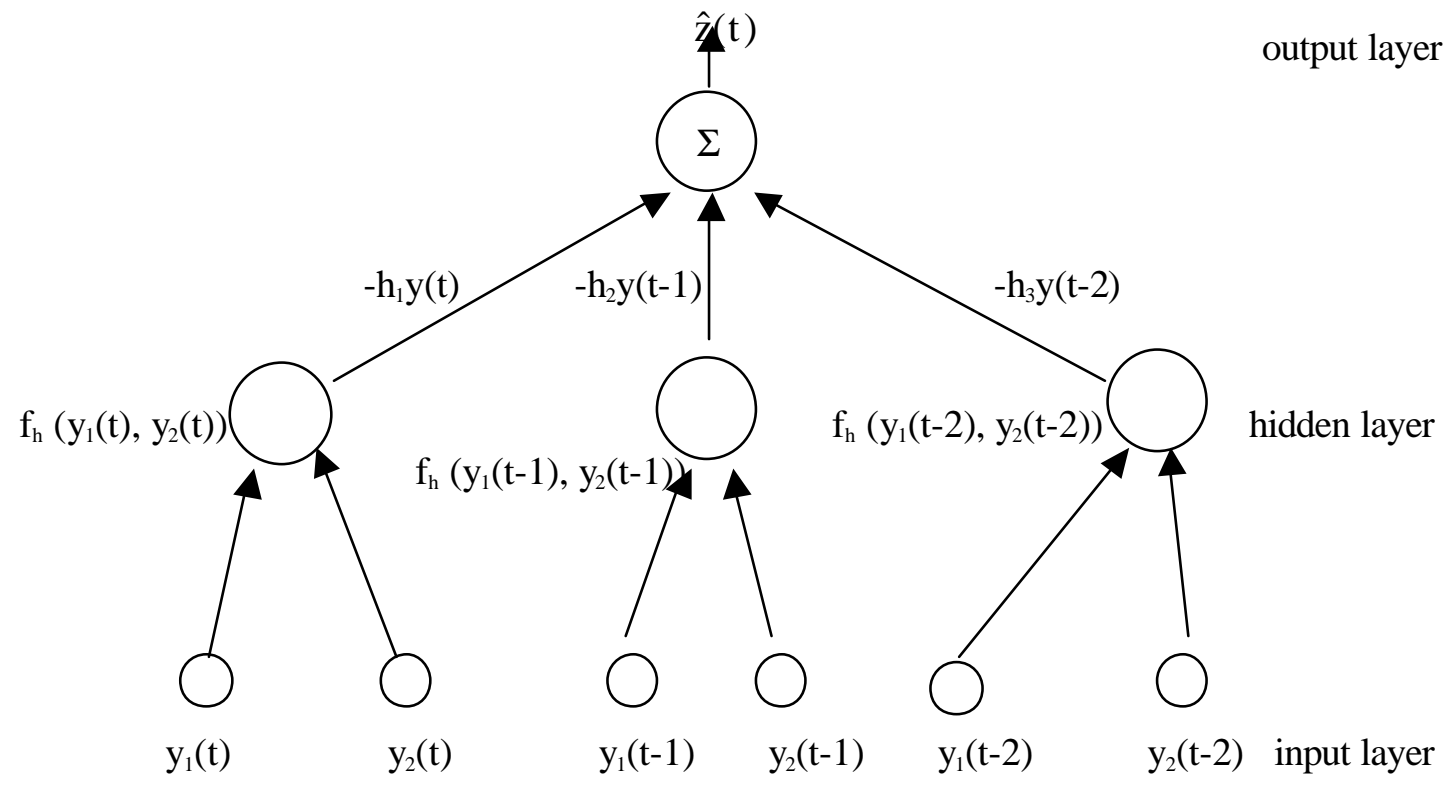

If $\mathrm{y}(\mathrm{t}-1)$ in (2.1) is missing, (2.1) becomes a subset VRDL model. In this case, the hidden unit operating $\mathrm{f}_{\mathrm{h}}\left(\mathrm{y}_{1}(\mathrm{t}-1), \mathrm{y}_{2}(\mathrm{t}-1)\right)$ becomes inoperative and the corresponding incoming and outgoing arcs become inhibitive. Analogously, Figure 3 illustrates the structure of a threelayered polynomial neural networks for the predictor of a VAR model

$$
\hat{y}(t)=-a_{1} y(t-1)-a_{2} y(t-2),
$$

where $a_{i}=\left[\begin{array}{llll}a_{1}(i) & a_{2}(i) & \cdots & a_{5}(i)\end{array}\right]^{\prime}, i=1$ and 2 .

Neural nets with inhibitor arcs and switchable connections are intuitively the most direct approach to increasing the modelling power of neural nets. These extensions provide neural nets with an ability to model sequentially changing time series systems with a subset structure. In the next section, we provide algorithms for both time and order updating which leads to the optimal synaptic weight updating and allows for the optimal dynamic node creation/deletion extended neural network. 
Figure 3: A three-layered polynomial neural network for a VAR model

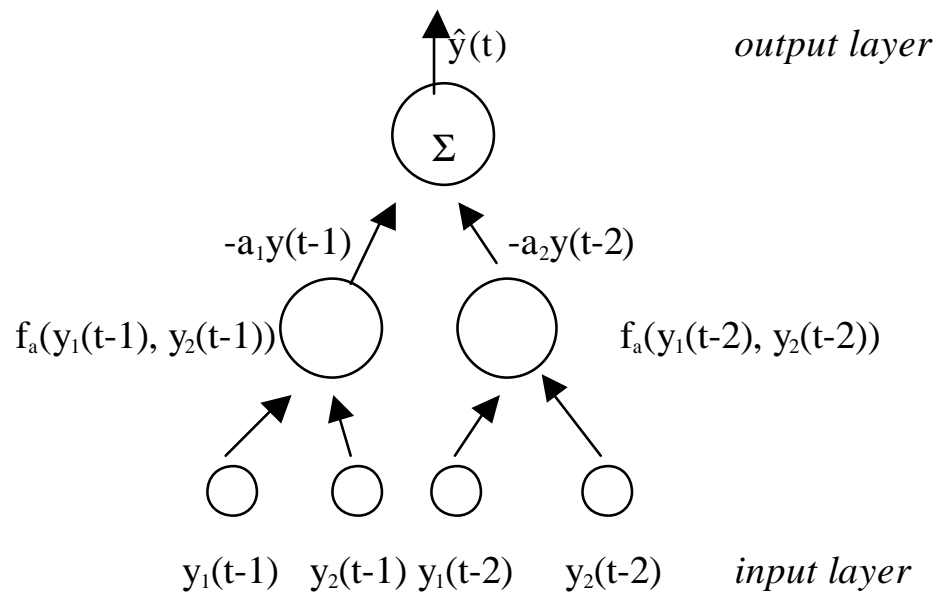

\section{THE LATTICE-LADDER ALGORITHM FOR SUBSET VAR AND VRDL}

\section{MODELLING}

In this section, we introduce a double (a-priori/a-posterior) lattice-ladder recursive algorithm for subset VRDL and VAR modelling. This is followed by providing the orderrecursive algorithm, which can be used to initialise the lattice-ladder algorithm.

If we let $\mathrm{z}(\mathrm{t})$ and $\mathrm{y}(\mathrm{t})$ be jointly stationary and zero mean vector time series, we can consider the subset VRDL(p) model of the form

$$
z(t)+\sum_{i=1}^{p} h_{i}^{\prime}\left(I_{s}\right) y(t+1-i)=\varepsilon^{h}(t)
$$

As defined in (1.2), $I_{s}$ represents an integer set with elements $i_{1}, i_{2}, \cdots, i_{s}$, $1 \leq \mathrm{i}_{1} \leq \cdots \leq \mathrm{i}_{\mathrm{s}} \leq \mathrm{p}-1 . \quad \mathrm{h}_{\mathrm{i}}^{\prime}\left(\mathrm{I}_{\mathrm{s}}\right)=0$, as $\mathrm{i} \in \mathrm{I}_{\mathrm{s}}$. The disturbance variance-covariance matrix is $\Omega\left(\mathrm{I}_{\mathrm{s}}\right)$ and the covariance matrix , so called partial correlation matrix, between $\varepsilon^{\mathrm{h}}(\mathrm{t})$ and $\mathrm{y}(\mathrm{t}-\mathrm{p})$ is $\Delta^{\mathrm{h}}\left(\mathrm{I}_{\mathrm{s}}\right)$. Given two finite data sample sets, $\{\mathrm{y}(1), \ldots, \mathrm{y}(\mathrm{T})\}$ and $\{\mathrm{z}(1), \ldots, \mathrm{z}(\mathrm{T})\}$, and both $y(j)=0$ and $z(j)=0$ for $j<1$, we would need to sequentially estimate all possible subset VRDL models from (3.1) using the pre-windowed case investigated by Penm et al (1995). Since the actual scheme of (3.1) may not be order $p$, we denote the resulting estimates of $h_{i}$ 
by $h_{p, T}(i)$, where $T$ is the sample size under examination. Then the predictor of (3.1) is of the form :

$$
\hat{\mathrm{z}}(\mathrm{i})=-\mathrm{H}_{\mathrm{p}, \mathrm{T}}^{\prime}\left(\mathrm{I}_{\mathrm{s}}\right) \mathrm{Y}_{\mathrm{p}, \mathrm{i}}\left(\mathrm{I}_{\mathrm{s}}\right) \text {, }
$$

where $\quad \mathrm{Y}_{\mathrm{p}, \mathrm{i}}=\left[\begin{array}{llll}\mathrm{y}^{\prime}(\mathrm{i}) & \mathrm{y}^{\prime}(\mathrm{i}-1) & \cdots & \mathrm{y}^{\prime}(\mathrm{i}-\mathrm{p}+1)\end{array}\right]^{\prime}, \mathrm{H}_{\mathrm{p}, \mathrm{T}}=\left[\begin{array}{llll}\mathrm{h}_{\mathrm{p}, \mathrm{T}}^{\prime}(1) & \mathrm{h}_{\mathrm{p}, \mathrm{T}}^{\prime}(2) & \cdots & \mathrm{h}_{\mathrm{p}, \mathrm{T}}^{\prime}(\mathrm{p})\end{array}\right]^{\prime}$. $H_{p, T}\left(I_{s}\right)$ and $Y_{p, i}\left(I_{s}\right)$ are formed by removing the $\left(i_{1}\right), \ldots,\left(i_{s}\right)$ th row block of $H_{p, T}$ and $Y_{p, i}$ respectively. The a-posterior prediction residual vector for observation $\mathrm{i}$ is defined as

$$
\varepsilon_{\mathrm{p}, \mathrm{i}}^{\mathrm{h}}\left(\mathrm{I}_{\mathrm{s}}\right)=\mathrm{z}(\mathrm{i})+\mathrm{H}_{\mathrm{p}, \mathrm{T}}^{\prime}\left(\mathrm{I}_{\mathrm{s}}\right) \mathrm{Y}_{\mathrm{p}, \mathrm{i}}\left(\mathrm{I}_{\mathrm{s}}\right)
$$

and the a-priori prediction residual vector for observation $\mathrm{i}$ is

$$
\mathrm{e}_{\mathrm{p}, \mathrm{i}}^{\mathrm{h}}\left(\mathrm{I}_{\mathrm{s}}\right)=\mathrm{z}(\mathrm{i})+\mathrm{H}_{\mathrm{p}, \mathrm{T}-1}^{\prime}\left(\mathrm{I}_{\mathrm{s}}\right) \mathrm{Y}_{\mathrm{p}, \mathrm{i}}\left(\mathrm{I}_{\mathrm{s}}\right)
$$

In reality, many time series systems present complex non-stationary features and cannot be modelled by assuming that $\mathrm{y}(\mathrm{t})$ and $\mathrm{z}(\mathrm{t})$ are stationary(see Bollerslev et al (1992)). Thus, an estimate of the structure at time t should give a higher weight to the more recent observations and a lower weight to the observations of the remote past. Thus, for a VRDL model fitted to these two sample sets using the pre-windowed method of forming the sample covariance matrix, we have

$$
\begin{aligned}
& \mathrm{R}_{\mathrm{p}-1, \mathrm{~T}}\left(\mathrm{I}_{\mathrm{s}}\right) \mathrm{H}_{\mathrm{p}, \mathrm{T}}\left(\mathrm{I}_{\mathrm{s}}\right)=-\mathrm{r}_{\mathrm{p}-1, \mathrm{~T}}\left(\mathrm{I}_{\mathrm{s}}\right) \text {, where } \mathrm{R}_{\mathrm{p}-1, \mathrm{~T}}\left(\mathrm{I}_{\mathrm{s}}\right)=\sum_{\mathrm{i}=1}^{\mathrm{T}} \lambda^{\mathrm{T}-\mathrm{i}} \mathrm{Y}_{\mathrm{p}, \mathrm{i}}\left(\mathrm{I}_{\mathrm{s}}\right) \mathrm{Y}_{\mathrm{p}, \mathrm{i}}^{\prime}\left(\mathrm{I}_{\mathrm{s}}\right) \\
& \mathrm{r}_{\mathrm{p}-1, \mathrm{~T}}\left(\mathrm{I}_{\mathrm{s}}\right)=\sum_{\mathrm{i}=1}^{\mathrm{T}} \lambda^{\mathrm{T}-\mathrm{i}} \mathrm{Y}_{\mathrm{p}, \mathrm{i}}\left(\mathrm{I}_{\mathrm{s}}\right) \mathrm{z}^{\prime}(\mathrm{i}) \text {, and } \Omega_{\mathrm{p}, \mathrm{T}}\left(\mathrm{I}_{\mathrm{s}}\right)=\sum_{\mathrm{i}=1}^{\mathrm{T}} \lambda^{\mathrm{T}-\mathrm{i}} \varepsilon_{\mathrm{p}, \mathrm{i}}^{\mathrm{h}}\left(\mathrm{I}_{\mathrm{s}}\right) \mathcal{E}_{\mathrm{p}, \mathrm{i}}^{\prime \mathrm{h}}\left(\mathrm{I}_{\mathrm{s}}\right)
\end{aligned}
$$

where $\lambda, 0<\lambda \leq 1$, is the forgetting factor as described in Hannan and Deistler (1988).

The forgetting factor is suitable for a model in which the underlying relationships between the variables involved change smoothly and gradually. When $\lambda=1$, the recursions are implemented in the stationary situation. 
Next we introduce a further regressor $y(t-p)$ into the VRDL model. For this enlarged $\operatorname{VRDL}\left(\mathrm{p}+1, \mathrm{I}_{\mathrm{s}}\right)$ model, we have

$$
R_{p, T}\left(I_{s}\right) H_{p+1, T}\left(I_{s}\right)=-\left[\begin{array}{c}
r_{p-1, T}\left(I_{s}\right) \\
v_{p, T}\left(I_{s}\right)
\end{array}\right],
$$

where $v_{p, T}\left(I_{s}\right)=\sum_{i=1}^{T} \lambda^{T-i} y(i-p) z^{\prime}(i)$

Following the work introduced by Carayannis et al (1986), we consider the forward subset VAR(p) model with the deleted elements $i_{1}, \dot{i}_{2}, \ldots, i_{s}$ of the form

$$
\sum_{i=0}^{p} a_{i}\left(I_{s}\right) y(t-i)=\varepsilon\left(t, I_{s}\right),\left\{a_{0}\left(I_{s}\right)=I, a_{i}\left(I_{s}\right)=0, \text { as } i \in I_{s},\right\}
$$

where $\mathrm{E}\left\{\varepsilon\left(\mathrm{t}, \mathrm{I}_{\mathrm{s}}\right)\right\}=0, \mathrm{E}\left\{\varepsilon\left(\mathrm{t}, \mathrm{I}_{\mathrm{s}}\right) \mathrm{y}^{\prime}(\mathrm{t}-\mathrm{p}-1)\right\}=\Delta\left(\mathrm{I}_{\mathrm{s}}\right)$ and $\mathrm{E}\left\{\varepsilon\left(\mathrm{t}, \mathrm{I}_{\mathrm{s}}\right) \varepsilon^{\prime}\left(\mathrm{t}, \mathrm{I}_{\mathrm{s}}\right)\right\}=\mathrm{V}\left(\mathrm{I}_{\mathrm{s}}\right)$ as $\mathrm{k}=0$;

$=0$ as $\mathrm{k}>0$. We also consider a backward subset $\operatorname{VAR}(\mathrm{p})$ model of the form

$$
\sum_{i=0}^{p} b_{i}\left(M_{s}\right) y(t-p+i)=\bar{\varepsilon}\left(t, M_{s}\right),\left\{b_{0}\left(M_{s}\right)=I, \quad b_{i}\left(M_{s}\right)=0, \text { as } i \in M_{s},\right\}
$$

where $M_{s}$ represents an integer set with elements $m_{1}, m_{2}, \ldots, m_{s}, m_{j}=p+1-i_{j}, j=1,2, \ldots, s$.

Suppose for the given sample $\{\mathrm{y}(1), \ldots, \mathrm{y}(\mathrm{T})\}$, we need to fit all possible subset VAR models from (3.4) and (3.5). Since the actual scheme may not be of order p, we denote the resulting estimates of $a_{k}$ and $b_{k}$ by $a_{p, T}(k)$ and $b_{p, T}(k)$ respectively. Then we define

$$
A_{p, T}^{\prime}=\left[a_{p, T}(1) \cdots a_{p, T}(p)\right], \quad B_{p, T}^{\prime}=\left[b_{p, T}(p) \cdots b_{p, T}(1)\right] .
$$

For a forward $\operatorname{VAR}\left(\mathrm{p}, \mathrm{I}_{\mathrm{s}}\right)$ fitted by this sample set, we have

$$
\begin{gathered}
R_{p, T}\left(I_{s}\right)=\sum_{i=1}^{T} \lambda^{T-i} Y_{p+1, i}\left(L_{s}\right) Y_{p+1, i}^{\prime}\left(L_{s}\right) \text {, where } Y_{p+1, i}\left(L_{s}\right)=\left[\begin{array}{c}
y(i) \\
Y_{p, i-1}\left(I_{s}\right)
\end{array}\right]=\left[\begin{array}{c}
Y_{p, i}\left(L_{s}\right) \\
y(i-p)
\end{array}\right], \\
R_{p, T}\left(I_{s}\right)\left[\begin{array}{c}
I \\
A_{p, T}\left(I_{s}\right)
\end{array}\right]=\left[\begin{array}{c}
V_{p, T}\left(I_{s}\right) \\
0
\end{array}\right], \varepsilon_{p, i}\left(I_{s}\right)=y(i)+A_{p, T}^{\prime}\left(I_{s}\right) Y_{p, i-1}\left(I_{s}\right)
\end{gathered}
$$


where $\mathrm{L}_{\mathrm{s}}$ represents an integer set with elements $l_{\mathrm{j}}, \mathrm{j}=1, \ldots, \mathrm{s}$, and $l_{\mathrm{j}}=\mathrm{i}_{\mathrm{j}}+1 . \mathrm{Y}_{\mathrm{p}+1, \mathrm{i}}\left(\mathrm{L}_{\mathrm{s}}\right)$ and $\mathrm{Y}_{\mathrm{p}, \mathrm{i}}\left(\mathrm{L}_{\mathrm{s}}\right)$ are formed by removing the $\left(l_{1}\right), \ldots,\left(l_{\mathrm{s}}\right)^{\prime}$ th row block of $\mathrm{Y}_{\mathrm{p}+1, \mathrm{i}}$ and $\mathrm{Y}_{\mathrm{p}, \mathrm{i}}$ respectively. Similarly $A_{p, T}\left(I_{s}\right)$ and $Y_{p, i-1}\left(I_{s}\right)$ are formed by removing the $\left(i_{1}\right), \ldots$, (i $\left.i_{s}\right)^{\prime}$ th row block of $A_{p, T}$ and $\mathrm{Y}_{\mathrm{p}, \mathrm{i}-\mathrm{1}}$ respectively. $\varepsilon_{\mathrm{p}, \mathrm{i}}\left(\mathrm{I}_{\mathrm{s}}\right)$ will be called the a-posterior forward AR residual vector to distinguish it from the a-prior forward $\mathrm{AR}$ residual vector defined as

$$
\mathrm{e}_{\mathrm{p}, \mathrm{i}}\left(\mathrm{I}_{\mathrm{s}}\right)=\mathrm{y}(\mathrm{i})+\mathrm{A}_{\mathrm{p}, \mathrm{T}-1}^{\prime}\left(\mathrm{I}_{\mathrm{s}}\right) \mathrm{Y}_{\mathrm{p}, \mathrm{i}-1}\left(\mathrm{I}_{\mathrm{s}}\right)
$$

In addition, for the corresponding backward $\operatorname{VAR}\left(\mathrm{p}, \mathrm{M}_{\mathrm{s}}\right)$, we have

$$
\begin{gathered}
R_{p, T}\left(M_{s}\right)=\sum_{i=1}^{T} \lambda^{T-i}\left[\begin{array}{c}
Y_{p, i}\left(M_{s}\right) \\
y(i-p)
\end{array}\right]\left[\begin{array}{ll}
Y_{p, i}^{\prime}\left(M_{s}\right) & y^{\prime}(i-p)
\end{array}\right]=\left[\begin{array}{cc}
D_{p, T}\left(M_{s}\right) & r_{p, T}^{b}\left(M_{s}\right) \\
r_{p, T}^{b}\left(M_{s}\right) & v_{p, T}^{b}\left(M_{s}\right)
\end{array}\right], \\
R_{p, T}\left(M_{s}\right)\left[\begin{array}{c}
B_{p, T}\left(M_{s}\right) \\
I
\end{array}\right]=\left[\begin{array}{c}
0 \\
\bar{V}_{p, T}\left(M_{s}\right)
\end{array}\right], \bar{\varepsilon}_{p, i}\left(M_{s}\right)=B_{p, T}^{\prime}\left(M_{s}\right) Y_{p, i}\left(M_{s}\right)+y(i-p),
\end{gathered}
$$

where $B_{p, T}\left(M_{s}\right)$ and $Y_{p, i}\left(M_{s}\right)$ are formed by removing the $\left(p+1-m_{1}\right), \ldots,\left(p+1-m_{s}\right)$ th row block of $B_{p, T}$ and $Y_{p, i}$ respectively. Thus $R_{p, T}\left(M_{s}\right)=R_{p, T}\left(I_{s}\right)$. Next, we define the associated VAR and VRDL partial correlation matrices of the form

$$
\begin{aligned}
& \Delta_{\mathrm{p}+1, \mathrm{~T}}\left(\mathrm{I}_{\mathrm{s}}\right)=v_{\mathrm{p}+1, \mathrm{~T}}^{\mathrm{b}}\left(\mathrm{M}_{\mathrm{s}}\right)+\mathrm{A}_{\mathrm{p}, \mathrm{T}}^{\prime}\left(\mathrm{I}_{\mathrm{s}}\right) \mathrm{r}_{\mathrm{p}, \mathrm{T}-1}^{\mathrm{b}}\left(\mathrm{M}_{\mathrm{s}}\right), \\
& \Delta_{\mathrm{p}+1, \mathrm{~T}}^{\mathrm{h}}\left(\mathrm{I}_{\mathrm{s}}\right)=v_{\mathrm{p}, \mathrm{T}}\left(\mathrm{I}_{\mathrm{s}}\right)+\mathrm{B}_{\mathrm{p}, \mathrm{T}}^{\prime}\left(\mathrm{M}_{\mathrm{s}}\right) \mathrm{r}_{\mathrm{p}-1, \mathrm{~T}}\left(\mathrm{I}_{\mathrm{s}}\right),
\end{aligned}
$$

the forward and backward reflection matrices of the form

$$
\begin{aligned}
& \mathrm{K}_{\mathrm{p}+1, \mathrm{~T}}^{\mathrm{f}}\left(\mathrm{I}_{\mathrm{s}}\right)=-\mathrm{V}_{\mathrm{p}, \mathrm{T}}^{-1}\left(\mathrm{I}_{\mathrm{s}}\right) \Delta_{\mathrm{p}+1, \mathrm{~T}}^{\prime}\left(\mathrm{I}_{\mathrm{s}}\right), \\
& \mathrm{K}_{\mathrm{p}+1, \mathrm{~T}}^{\mathrm{b}}\left(\mathrm{M}_{\mathrm{s}}\right)=-\overline{\mathrm{V}}_{\mathrm{p}, \mathrm{T}-1}^{-1}\left(\mathrm{M}_{\mathrm{s}}\right) \Delta_{\mathrm{p}+1, \mathrm{~T}}\left(\mathrm{I}_{\mathrm{s}}\right),
\end{aligned}
$$

and the ladder gain matrix of the form

$$
\mathrm{K}_{\mathrm{p}+1, \mathrm{~T}}\left(\mathrm{I}_{\mathrm{s}}\right)=-\overline{\mathrm{V}}_{\mathrm{p}, \mathrm{T}}^{-1}\left(\mathrm{M}_{\mathrm{s}}\right) \Delta_{\mathrm{p}+1, \mathrm{~T}}^{\mathrm{h}}\left(\mathrm{I}_{\mathrm{s}}\right) .
$$

In addition, $\bar{\varepsilon}_{\mathrm{p}, \mathrm{i}}\left(\mathrm{M}_{\mathrm{s}}\right)$ is called the a-posterior backward $\mathrm{AR}$ residual vector to distinguish it from the a-prior backward AR residual vector as defined by 


$$
\overline{\mathrm{e}}_{\mathrm{p}, \mathrm{i}}\left(\mathrm{M}_{\mathrm{s}}\right)=\left[\mathrm{B}_{\mathrm{p}, \mathrm{T}-1}^{\prime}\left(\mathrm{M}_{\mathrm{s}}\right) \mathrm{I}\right]\left[\begin{array}{c}
\mathrm{Y}_{\mathrm{p}, \mathrm{i}}\left(\mathrm{M}_{\mathrm{s}}\right) \\
\mathrm{y}(\mathrm{i}-\mathrm{p})
\end{array}\right]
$$

A double (a-priori/a-posterior) lattice recursive algorithm for subset VRDL and VAR modelling can then be developed as follows: ${ }^{2}$

Lattice algorithm for subset VAR models

$$
\begin{aligned}
& \mathrm{e}_{\mathrm{p}+1, \mathrm{~T}}\left(\mathrm{I}_{\mathrm{s}}\right)=\mathrm{e}_{\mathrm{p}, \mathrm{T}}\left(\mathrm{I}_{\mathrm{s}}\right)+\mathrm{K}_{\mathrm{p}+1, \mathrm{~T}-1}^{\prime \mathrm{b}}\left(\mathrm{M}_{\mathrm{s}}\right) \overline{\mathrm{e}}_{\mathrm{p}, \mathrm{T}-1}\left(\mathrm{M}_{\mathrm{s}}\right) \\
& \overline{\mathrm{e}}_{\mathrm{p}+1, \mathrm{~T}}\left(\mathrm{M}_{\mathrm{s}}\right)=\overline{\mathrm{e}}_{\mathrm{p}, \mathrm{T}-1}\left(\mathrm{M}_{\mathrm{s}}\right)+\mathrm{K}_{\mathrm{p}+1, \mathrm{~T}-1}^{\prime \mathrm{f}}\left(\mathrm{I}_{\mathrm{s}}\right) \mathrm{e}_{\mathrm{p}, \mathrm{T}}\left(\mathrm{I}_{\mathrm{s}}\right) \\
& \Delta_{\mathrm{p}+1, \mathrm{~T}}\left(\mathrm{I}_{\mathrm{s}}\right)=\lambda \Delta_{\mathrm{p}+1, \mathrm{~T}-1}\left(\mathrm{I}_{\mathrm{s}}\right)+\bar{\varepsilon}_{\mathrm{p}, \mathrm{T}-1}\left(\mathrm{M}_{\mathrm{s}}\right) \mathrm{e}_{\mathrm{p}, \mathrm{T}}^{\prime}\left(\mathrm{I}_{\mathrm{s}}\right) \\
& \mathrm{K}_{\mathrm{p}+1, \mathrm{~T}}^{\mathrm{f}}\left(\mathrm{I}_{\mathrm{s}}\right)=-\mathrm{V}_{\mathrm{p}, \mathrm{T}}^{-1}\left(\mathrm{I}_{\mathrm{s}}\right) \Delta_{\mathrm{p}+1, \mathrm{~T}}^{\prime}\left(\mathrm{I}_{\mathrm{s}}\right) \\
& \mathrm{K}_{\mathrm{p}+1, \mathrm{~T}}^{\mathrm{b}}\left(\mathrm{M}_{\mathrm{s}}\right)=-\overline{\mathrm{V}}_{\mathrm{p}, \mathrm{T}-1}^{-1}\left(\mathrm{M}_{\mathrm{s}}\right) \Delta_{\mathrm{p}+1, \mathrm{~T}}\left(\mathrm{I}_{\mathrm{s}}\right) \\
& \varepsilon_{\mathrm{p}+1, \mathrm{~T}}\left(\mathrm{I}_{\mathrm{s}}\right)=\varepsilon_{\mathrm{p}, \mathrm{T}}\left(\mathrm{I}_{\mathrm{s}}\right)+\mathrm{K}_{\mathrm{p}+1, \mathrm{~T}}^{\mathrm{b}}\left(\mathrm{M}_{\mathrm{s}}\right) \bar{\varepsilon}_{\mathrm{p}, \mathrm{T}-1}\left(\mathrm{M}_{\mathrm{s}}\right) \\
& \bar{\varepsilon}_{\mathrm{p}+1, \mathrm{~T}}\left(\mathrm{M}_{\mathrm{s}}\right)=\bar{\varepsilon}_{\mathrm{p}, \mathrm{T}-1}\left(\mathrm{M}_{\mathrm{s}}\right)+\mathrm{K}_{\mathrm{p}+1, \mathrm{~T}}^{\prime \mathrm{f}}\left(\mathrm{I}_{\mathrm{s}}\right) \varepsilon_{\mathrm{p}, \mathrm{T}}\left(\mathrm{I}_{\mathrm{s}}\right) \\
& \overline{\mathrm{V}}_{\mathrm{p}+1, \mathrm{~T}}\left(\mathrm{M}_{\mathrm{s}}\right)=\lambda \overline{\mathrm{V}}_{\mathrm{p}+1, \mathrm{~T}-1}\left(\mathrm{M}_{\mathrm{s}}\right)+\bar{\varepsilon}_{\mathrm{p}+1, \mathrm{~T}}\left(\mathrm{M}_{\mathrm{s}}\right) \overline{\mathrm{e}}_{\mathrm{p}+1, \mathrm{~T}}^{\prime}\left(\mathrm{M}_{\mathrm{s}}\right) \\
& \mathrm{V}_{\mathrm{p}+1, \mathrm{~T}}\left(\mathrm{I}_{\mathrm{s}}\right)=\lambda \mathrm{V}_{\mathrm{p}+1, \mathrm{~T}-1}\left(\mathrm{I}_{\mathrm{s}}\right)+\varepsilon_{\mathrm{p}+1, \mathrm{~T}}\left(\mathrm{I}_{\mathrm{s}}\right) \mathrm{e}_{\mathrm{p}+1, \mathrm{~T}}^{\prime}\left(\mathrm{I}_{\mathrm{s}}\right)
\end{aligned}
$$

Ladder algorithm for subset VRDL models:

$$
\begin{aligned}
& \mathrm{e}_{\mathrm{p}+1, \mathrm{~T}}^{\mathrm{h}}\left(\mathrm{I}_{\mathrm{s}}\right)=\mathrm{e}_{\mathrm{p}, \mathrm{T}}^{\mathrm{h}}\left(\mathrm{I}_{\mathrm{s}}\right)+\mathrm{K}_{\mathrm{p}+1, \mathrm{~T}-1}^{\prime}\left(\mathrm{I}_{\mathrm{s}}\right) \overline{\mathrm{e}}_{\mathrm{p}, \mathrm{T}}\left(\mathrm{M}_{\mathrm{s}}\right) \\
& \Delta_{\mathrm{p}+1, \mathrm{~T}}^{\mathrm{h}}\left(\mathrm{I}_{\mathrm{s}}\right)=\lambda \Delta_{\mathrm{p}+1, \mathrm{~T}-1}^{\mathrm{h}}\left(\mathrm{I}_{\mathrm{s}}\right)+\bar{\varepsilon}_{\mathrm{p}, \mathrm{T}}\left(\mathrm{M}_{\mathrm{s}}\right) \mathrm{e}_{\mathrm{p}, \mathrm{T}}^{\mathrm{h}}\left(\mathrm{I}_{\mathrm{s}}\right) \\
& \mathrm{K}_{\mathrm{p}+1, \mathrm{~T}}\left(\mathrm{I}_{\mathrm{s}}\right)=-\overline{\mathrm{V}}_{\mathrm{p}, \mathrm{T}}^{-1}\left(\mathrm{M}_{\mathrm{s}}\right) \Delta_{\mathrm{p}+1, \mathrm{~T}}^{\mathrm{h}}\left(\mathrm{I}_{\mathrm{s}}\right) \\
& \varepsilon_{\mathrm{p}+1, \mathrm{~T}}^{\mathrm{h}}\left(\mathrm{I}_{\mathrm{s}}\right)=\varepsilon_{\mathrm{p}, \mathrm{T}}^{\mathrm{h}}\left(\mathrm{I}_{\mathrm{s}}\right)+\mathrm{K}_{\mathrm{p}+1, \mathrm{~T}}^{\prime}\left(\mathrm{I}_{\mathrm{s}}\right) \bar{\varepsilon}_{\mathrm{p}, \mathrm{T}}\left(\mathrm{M}_{\mathrm{s}}\right) \\
& \Omega_{\mathrm{p}+1, \mathrm{~T}}\left(\mathrm{I}_{\mathrm{s}}\right)=\lambda \Omega_{\mathrm{p}+1, \mathrm{~T}-1}\left(\mathrm{I}_{\mathrm{s}}\right)+\varepsilon_{\mathrm{p}+1, \mathrm{~T}}^{\mathrm{h}}\left(\mathrm{I}_{\mathrm{s}}\right) \mathrm{e}_{\mathrm{p}+1, \mathrm{~T}}^{\mathrm{h}}\left(\mathrm{I}_{\mathrm{s}}\right)
\end{aligned}
$$

To initialise the lattice-ladder algorithm, we extend the order update algorithm proposed by Penm and Terrell (1982) and incorporate it in the current overall algorithm. If we let $\mathrm{q}$

\footnotetext{
2 For brevity, proofs are not presented, but they are available upon request.
} 
denote the number of non-zero coefficient matrices of the VAR model, we have the following order-update equations:

Order updating for subset VAR models

$$
\begin{aligned}
& \Delta=v_{\mathrm{p}+1, \mathrm{~T}}^{\mathrm{b}}\left(\mathrm{M}_{\mathrm{s}}\right)+\mathrm{A}_{\mathrm{p}, \mathrm{T}}^{\prime}\left(\mathrm{I}_{\mathrm{s}}\right) \mathrm{r}_{\mathrm{p}, \mathrm{T}-1}^{\mathrm{b}}\left(\mathrm{M}_{\mathrm{s}}\right) \\
& \mathrm{K}_{\mathrm{p}+1, \mathrm{~T}}^{\mathrm{f}}\left(\mathrm{I}_{\mathrm{s}}\right)=-\mathrm{V}_{\mathrm{p}, \mathrm{T}}^{-1}\left(\mathrm{I}_{\mathrm{s}}\right) \Delta^{\prime} \\
& \mathrm{K}_{\mathrm{p}+1, \mathrm{~T}}^{\mathrm{b}}\left(\mathrm{M}_{\mathrm{s}}\right)=-\overline{\mathrm{V}}_{\mathrm{p}, \mathrm{T}-1}^{-1}\left(\mathrm{M}_{\mathrm{s}}\right) \Delta \\
& \mathrm{A}_{\mathrm{p}+1, \mathrm{~T}}\left(\mathrm{I}_{\mathrm{s}}\right)=\left[\begin{array}{c}
\mathrm{A}_{\mathrm{p}, \mathrm{T}}\left(\mathrm{I}_{\mathrm{s}}\right) \\
0
\end{array}\right]+\left[\begin{array}{c}
\mathrm{B}_{\mathrm{p}, \mathrm{T}-1}\left(\mathrm{M}_{\mathrm{s}}\right) \\
\mathrm{I}
\end{array}\right] \mathrm{K}_{\mathrm{p}+1, \mathrm{~T}}^{\mathrm{b}}\left(\mathrm{M}_{\mathrm{s}}\right) \\
& \mathrm{B}_{\mathrm{p}+1, \mathrm{~T}}\left(\mathrm{M}_{\mathrm{s}}\right)=\left[\begin{array}{c}
0 \\
\mathrm{~B}_{\mathrm{p}, \mathrm{T}-1}\left(\mathrm{M}_{\mathrm{s}}\right)
\end{array}\right]+\left[\begin{array}{c}
\mathrm{I} \\
\mathrm{A}_{\mathrm{p}, \mathrm{T}}\left(\mathrm{I}_{\mathrm{s}}\right)
\end{array}\right] \mathrm{K}_{\mathrm{p}+1, \mathrm{~T}}^{\mathrm{f}}\left(\mathrm{I}_{\mathrm{s}}\right) \\
& \overline{\mathrm{V}}_{\mathrm{p}+1, \mathrm{~T}}\left(\mathrm{M}_{\mathrm{s}}\right)=\overline{\mathrm{V}}_{\mathrm{p}, \mathrm{T}-1}\left(\mathrm{M}_{\mathrm{s}}\right)+\Delta \mathrm{K}_{\mathrm{p}+1, \mathrm{~T}}^{\mathrm{f}}\left(\mathrm{I}_{\mathrm{s}}\right) \\
& \mathrm{V}_{\mathrm{p}+1, \mathrm{~T}}\left(\mathrm{I}_{\mathrm{s}}\right)=\mathrm{V}_{\mathrm{p}, \mathrm{T}}\left(\mathrm{I}_{\mathrm{s}}\right)+\Delta^{\prime} \mathrm{K}_{\mathrm{p}+1, \mathrm{~T}}^{\mathrm{b}}\left(\mathrm{M}_{\mathrm{s}}\right)
\end{aligned}
$$

Order updating for subset VRDL models

$$
\begin{aligned}
& \Delta_{\mathrm{p}+1, \mathrm{~T}}^{\mathrm{h}}\left(\mathrm{I}_{\mathrm{s}}\right)=v_{\mathrm{p}, \mathrm{T}}\left(\mathrm{I}_{\mathrm{s}}\right)+\mathrm{B}_{\mathrm{p}, \mathrm{T}}^{\prime}\left(\mathrm{M}_{\mathrm{s}}\right) \mathrm{r}_{\mathrm{p}-1, \mathrm{~T}}\left(\mathrm{I}_{\mathrm{s}}\right) \\
& \mathrm{K}_{\mathrm{p}+1, \mathrm{~T}}\left(\mathrm{I}_{\mathrm{s}}\right)=-\overline{\mathrm{V}}_{\mathrm{p}, \mathrm{T}}^{-1}\left(\mathrm{M}_{\mathrm{s}}\right) \Delta_{\mathrm{p}+1, \mathrm{~T}}^{\mathrm{h}}\left(\mathrm{I}_{\mathrm{s}}\right) \\
& \mathrm{H}_{\mathrm{p}+1, \mathrm{~T}}\left(\mathrm{I}_{\mathrm{s}}\right)=\left[\begin{array}{c}
\mathrm{H}_{\mathrm{p}, \mathrm{T}}\left(\mathrm{I}_{\mathrm{s}}\right) \\
0
\end{array}\right]+\left[\begin{array}{c}
\mathrm{B}_{\mathrm{p}, \mathrm{T}}\left(\mathrm{M}_{\mathrm{s}}\right) \\
\mathrm{I}
\end{array}\right] \mathrm{K}_{\mathrm{p}+1, \mathrm{~T}}\left(\mathrm{I}_{\mathrm{s}}\right) \\
& \Omega_{\mathrm{p}+1, \mathrm{~T}}\left(\mathrm{I}_{\mathrm{s}}\right)=\Omega_{\mathrm{p}, \mathrm{T}}\left(\mathrm{I}_{\mathrm{s}}\right)+\Delta_{\mathrm{p}+1, \mathrm{~T}}^{\mathrm{h}}\left(\mathrm{I}_{\mathrm{s}}\right) \mathrm{K}_{\mathrm{p}+1, \mathrm{~T}}\left(\mathrm{I}_{\mathrm{s}}\right)
\end{aligned}
$$

In the special case, where the consecutive coefficient matrices $\mathrm{a}_{\mathrm{p}-\mathrm{k}}$ for the lags of $\mathrm{y}(\mathrm{t}-\mathrm{p}+\mathrm{k}), \mathrm{k}=0,1, \ldots, \mathrm{c}(\mathrm{c}<\mathrm{p}-1)$ of the forward VAR(p) model, fitted using the sample $\{\mathrm{y}(1), \ldots$, $\mathrm{y}(\mathrm{T})\}$, are missing, the estimated coefficient matrices are null, i.e. $\mathrm{a}_{\mathrm{p}-\mathrm{k}, \mathrm{T}}\left(\mathrm{I}_{\mathrm{s}}\right)=0$. The corresponding coefficient matrices, a-prior and a-posterior forward prediction residual vectors, and $\mathrm{V}$ of the forward $\mathrm{VAR}(\mathrm{p}-\mathrm{c}-1)$ model are sufficient to continue the recursive estimations. 
Similarly, if the consecutive coefficient matrices $h_{-k}$ of the VRDL(p) model are missing, the analogous relations will hold.

Again, in the special case where the consecutive coefficient matrices $\mathrm{b}_{\mathrm{p}-\mathrm{k}} \mathrm{k}=0,1, \ldots, \mathrm{d}$ $(\mathrm{d}<\mathrm{p}-1)$ of the backward $\operatorname{VAR}(\mathrm{p})$ model, fitted using the sample $\{\mathrm{y}(1), \ldots, \mathrm{y}(\mathrm{T})\}$, are missing, the coefficient matrices, a-prior and a-posterior backward prediction residual vectors, and $\overline{\mathrm{V}}$ from the backward VAR(p-d-1) fitted using the sample $\{\mathrm{y}(1), \ldots, \mathrm{y}(\mathrm{T}-\mathrm{d}-1)\}$ allow the proposed lattice-ladder and order-update recursions.

The order update algorithm is well suited to the initial stage of the lattice-ladder recursive algorithm. After the initialisation is carried out and all necessary lattice-ladder parameter matrices at $\mathrm{t}=\mathrm{T}$ are obtained, the lattice-ladder recursive algorithm can be carried out from $\mathrm{T}$ to $\mathrm{T}+1, \mathrm{~T}+2$, and so on. A model selection criterion is then used to select the lag structure of the optimal subset VRDL and that of the optimal subset VAR at each time instant. Finally, the associated coefficient matrices $\mathrm{H}_{i}\left(\mathrm{I}_{\mathrm{s}}\right)$ and $\mathrm{A}_{\mathrm{i}}\left(\mathrm{I}_{\mathrm{s}}\right)$ can be estimated. A detailed illustration for $\mathrm{K}=\mathrm{Q}=4$ is provided in Section 4 .

\section{ILLUSTRATION}

Suppose we wish to fit all possible subset VAR models for $\mathrm{y}(\mathrm{t})=\left\{\mathrm{y}_{1}, \mathrm{y}_{2}, \ldots, \mathrm{y}_{81}, \ldots\right\}$ up to and including the maximum lag $K=4$, and all possible subset VRDL systems, $z(t)=\left\{z_{1}, z_{2}, \ldots\right.$, $\left.\mathrm{z}_{81}, \ldots\right\}$ and $\mathrm{y}(\mathrm{t})=\left\{\mathrm{y}_{1}, \mathrm{y}_{2}, \ldots, \mathrm{y}_{81}, \ldots\right\}$, up to and including the maximum lag $\mathrm{Q}=\mathrm{K}$ at $\mathrm{T}=79,80$ 81 and so on. The following description would present a suitable method. Note that we use numerals $0,1,2$ to represent particular lags for $y(t-s), s \geq 0$ and underlined numerals $\underline{1}, \underline{2}, \ldots$ to represent particular leads for $\mathrm{y}(\mathrm{t}+\mathrm{s}), \mathrm{s} \geq 1$, in VAR models, and italic numerals $\mathbf{0 , 1 , 2}$ to represent particular lags for $\mathrm{y}(\mathrm{t}-\mathrm{s}), \mathrm{s} \geq 0$ in VRDL systems. 
In the initial stage, the parameter matrices $A_{p, T}, V_{p, T}, B_{p, T}, \bar{V}_{p, T}$ for each necessary subset VAR model and $\mathrm{H}_{\mathrm{p}, \mathrm{T}}$ and $\Omega_{\mathrm{p}, \mathrm{T}}$ for each necessary subset VRDL model can be obtained using the order update recursions at $\mathrm{T}=76,77,78$ and 79 . All necessary models for performing the order update recursions are listed in Table 1. To achieve this end, $\mathrm{K}$ should be set at 0 in the first recursion for each subset model at $\mathrm{T}=76$ (i.e. $79-4+1$ ), be set at 1 in the second recursion at $\mathrm{T}=77$, and then increase linearly in time until it reaches 4 at $\mathrm{T}=79$. Next, we carry out the lattice-ladder recursions from $\mathrm{T}=79$ to 80,81 , and so on. A procedure for performing the order update recursions is as follows:

(1) All one-lag forward and backward VAR models $(1, \underline{1}),(2, \underline{2}),(3, \underline{3})$, and $(4, \underline{4})$ at $\mathrm{T}=76$, 77, 78 and 79 and all one-lag VRDL models $0,1,2$ at $\mathrm{T}=79$ must be fitted to start the recursions.

(2) Carry at the order update recursions to fit all 2-lag forward and backward VAR models at $\mathrm{T}=77,78,79$. All estimates of each 2lag VRDL system at $\mathrm{T}=79$ can also be obtained. Stage 1 in Table 1 presents the required models for performing the recursions.

(3) Again, carry out the order update recursions to estimate all 3-lag subset VAR models at $\mathrm{T}=78$ and 79 and VRDL systems at $\mathrm{T}=79$. Stage 2 in Table 1 illustrates this situation.

(4) Obtain the estimates of the full order forward and backward VAR models with $K=4$ and the full order VRDL with $\mathrm{Q}=\mathrm{K}$ at $\mathrm{T}=79$ using the order update recursions. Stage 3 in Table 1 lists all models required.

To this stage, both $\Delta$ for each subset VAR and $\Delta^{\mathrm{h}}$ for each subset VRDL are obtained. Assuming that all relevant quantities up to and including time $\mathrm{T}$ are known, the procedure for carrying out the lattice-ladder recursions from $\mathrm{T}$ to $\mathrm{T}+1$ is as follows:

Step 1: To begin with, all 1-lag forward and backward VAR models and all 1-lag VRDL models must be fitted. We then compute the associated prediction residual vectors 
$\mathrm{e}_{\mathrm{p}, \mathrm{T}}^{\mathrm{h}}\left(\mathrm{I}_{\mathrm{s}}\right), \bar{\varepsilon}_{\mathrm{p}, \mathrm{T}}^{\mathrm{h}}\left(\mathrm{I}_{\mathrm{s}}\right), \mathrm{e}_{\mathrm{p}, \mathrm{T}}\left(\mathrm{I}_{\mathrm{s}}\right), \bar{\varepsilon}_{\mathrm{p}, \mathrm{T}}\left(\mathrm{I}_{\mathrm{s}}\right), \mathrm{e}_{\mathrm{p}, \mathrm{T}}\left(\mathrm{M}_{\mathrm{s}}\right), \bar{\varepsilon}_{\mathrm{p}, \mathrm{T}}\left(\mathrm{M}_{\mathrm{s}}\right)$

by using the equations (3.3), (3.2), (3.7), (3.4), (3.9) and (3.5) respectively.

Step 2: For all 2-lag models, $\mathrm{e}_{\mathrm{p}+1, \mathrm{~T}+1}\left(\mathrm{I}_{\mathrm{s}}\right), \overline{\mathrm{e}}_{\mathrm{p}+1, \mathrm{~T}+1}\left(\mathrm{M}_{\mathrm{s}}\right), \mathrm{e}_{\mathrm{p}+1, \mathrm{~T}+1}^{\mathrm{h}}\left(\mathrm{I}_{\mathrm{s}}\right), \mathrm{p}=1$ can be obtained using (3.10.a), (3.10.b) and (3.10.j).

Step 3: The partial correlation, reflection coefficient and ladder gain matrices can be updated using (3.10.c), (3.10.d), (3.10.e), (3.10.k) and (3.10.1).

Step 4: We then use (3.10.f), (3.10.g) and (3.10.m) to estimate

$\varepsilon_{\mathrm{p}+1, \mathrm{~T}+1}\left(\mathrm{I}_{\mathrm{s}}\right), \bar{\varepsilon}_{\mathrm{p}+1, \mathrm{~T}+1}\left(\mathrm{M}_{\mathrm{s}}\right), \varepsilon_{\mathrm{p}+1, \mathrm{~T}+1}^{\mathrm{h}}\left(\mathrm{I}_{\mathrm{s}}\right), \mathrm{p}=1$.

Step 5: The residual variance-covariance matrices can be estimated using (3.10.h), (3.10.i) and (3.10.n).

Step 6: Repeat Steps 2 to 5 for all 3-lag models, and then all 4lag models.

Furthermore, an order selection criterion for the vector case as suggested by Hannan and Deistler (1988), could be modified to use at each time instant with the proposed method to select the optimal subset VAR and VRDL models. From now on, we will use MHQC as an abbreviation of the modified criterion, which is defined by

$$
\text { MHQC }=\log |\hat{\mathrm{G}}|+[2 \log \log f(\mathrm{~T}) / \mathrm{f}(\mathrm{T})] \mathrm{S}, \mathrm{f}(\mathrm{T})=\sum_{\mathrm{i}=1}^{\mathrm{T}} \lambda^{\mathrm{T}-\mathrm{i}},
$$

where $f(T)$ is the effective sample size, $S$ is the number of functionally independent parameters, and $\hat{G}$ is the estimate of $\mathrm{V}_{\mathrm{p}, \mathrm{T}}\left(\mathrm{I}_{\mathrm{s}}\right)$ for the VAR and the estimate of $\Omega_{\mathrm{p}, \mathrm{T}}\left(\mathrm{I}_{\mathrm{s}}\right)$ for the VRDL. 


\section{TABLE 1}

The necessary VRDLs and forward and backward VARs required to estimate all 2,3, or 4-lag subsets at $\mathrm{T}=79$, using the order update recursions, and to update their a-priori/a-posterior errors, using the double(a-priori/a-posterior)lattice-ladder structure.

\begin{tabular}{cccccc}
\hline \multicolumn{2}{c}{$\begin{array}{c}\text { the }(\mathrm{p}+1) \text { th model } \\
\text { T }\end{array}$} & \multicolumn{3}{c}{$\mathrm{T}$} & $\mathrm{T}$ \\
\hline Stage 1 & & & & \\
12 & $(79)$ & 1 & $(79)$ & $\underline{1}$ & $(78)$ \\
13 & $(79)$ & 1 & $(79)$ & $\underline{2}$ & $(78)$ \\
14 & $(79)$ & 1 & $(79)$ & $\underline{3}$ & $(78)$ \\
23 & $(79)$ & 2 & $(79)$ & $\underline{1}$ & $(77)$ \\
24 & $(79)$ & 2 & $(79)$ & $\underline{2}$ & $(77)$ \\
34 & $(79)$ & 3 & $(79)$ & $\underline{1}$ & $(76)$ \\
$\underline{12}$ & $(79)$ & 1 & $(79)$ & $\underline{1}$ & $(78)$ \\
$\underline{13}$ & $(79)$ & 2 & $(79)$ & $\underline{1}$ & $(77)$ \\
$\underline{14}$ & $(79)$ & 3 & $(79)$ & $\underline{1}$ & $(76)$ \\
$\underline{23}$ & $(79)$ & 1 & $(79)$ & $\underline{2}$ & $(78)$ \\
$\underline{24}$ & $(79)$ & 2 & $(79)$ & $\underline{2}$ & $(77)$ \\
$\underline{34}$ & $(79)$ & 1 & $(79)$ & $\underline{3}$ & $(78)$ \\
$\mathbf{0 1}$ & $(79)$ & & & $\underline{1}$ & $(79)$ \\
$\mathbf{0 2}$ & $(79)$ & $\mathbf{0}$ & $(79)$ & $\underline{2}$ & $(79)$ \\
$\mathbf{0 3}$ & $(79)$ & $\mathbf{0}$ & $(79)$ & $\underline{3}$ & $(79)$ \\
$\mathbf{1 2}$ & $(79)$ & $\mathbf{0}$ & $(79)$ & $\underline{1}$ & $(78)$ \\
$\mathbf{1 3}$ & $(79)$ & $\mathbf{1}$ & $(79)$ & $\underline{1}$ & $(77)$ \\
$\mathbf{2 3}$ & $(79)$ & $\mathbf{1}$ & $(79)$ & $(79)$ &
\end{tabular}

Stage 2

$\begin{array}{ll}123 & (79) \\ 124 & (79) \\ 134 & (79) \\ 234 & (79) \\ \underline{123} & (79) \\ \underline{124} & (79) \\ \underline{134} & (79) \\ \underline{234} & (79)\end{array}$

$12(79)$

$12(78)$

12 (79)

$\underline{23}(78)$

13 (79)

$\underline{13}(78)$

23 (79)

$\underline{12}$ (77)

12 (79)

$\frac{12}{12}$ (78)

$23 \quad$ (79)

$\underline{12}$ (77)

13 (79)

$\underline{13}$ (78)

12 (79)

$\underline{23}$ (78)

$012 \quad$ (79)

$01 \quad$ (79)

$\underline{12}$ (79)

$013 \quad$ (79)

023 (79)

$01 \quad$ (79)

23 (79)

123 (79)

02 (79)

$\underline{13}$ (79)

12 (79)

$\underline{12}$ (78)

Stage 3

\begin{tabular}{ll}
1234 & $(79)$ \\
1234 & $(79)$ \\
\hline $\mathbf{0 1 2 3}$ & $(79)$
\end{tabular}

$123 \quad$ (79)

$\underline{123}(78)$

$123 \quad$ (79)

$\underline{123}$ (78)

012 (79)

$\underline{123}$ (79) 
It is inappropriate to neglect possible zero constraints in the non-zero coefficient matrices of the optimal subset VAR and VRDL models. Geweke and Singleton (1981) introduced prior zero constraints in the coefficient matrices of a VRDL model to test hypotheses on the relations between observed economic time series and latent factors. Our experience (see Penm and Terrell (1984) and Penm et al (1992)) shows that the proposed search algorithm can, with minor modification, be adapted to select the optimum subset VAR and VRDL models with zero constraints using the prewindowed case.

\section{APPLICATIONS}

In this section, we present two applications to illustrate the practical use of the algorithm. The first application concerns a causality relationship between the stock and futures markets. The second application concerns the relationship between prices on an individual share and the underlying stock market.

For the first application, the extended two-layered neural network described in section 2 is used to model the relationship between the Australian stock and futures markets. In Australia, the main stock market indicator is the All Ordinaries Index (AOI). This index comprises around 320 of the largest stocks, is value-weighted and represents about $90-95 \%$ of total market capitalisation. The index is calculated on the basis of market capitalisation of the constituent stocks traded on the Australian Stock Exchange. The Sydney Futures Exchange trades a futures contract on the AOI. This contract is available on a quarterly expiry date and is known as the Share Price Index (SPI) Futures Contract. 
There is already a considerable literature examining the relationship between stock and futures market prices. The literature has either examined theoretical relationships between the markets through models such as the cost-of-carry (Chung (1991), MacKinlay and Ramaswamy (1988), Brailsford and Hodgson (1997)), or examined the causality between the markets through lead-lag relationships, cointegration tests or bivariate spillover models (Chan (1992), Chan et al (1991), Martens et al (1996), Tse (1995)). The general findings confirm a strong causality between the markets. This relationship is not unexpected given the pricing relationship between the markets and the fact that the basis reduces to zero at the maturity of the futures contract. However, there has been debate about the direction of causality with the evidence generally indicating that the futures market leads the stock market.

In this paper, data on the AOI and SPI are sampled daily between 27 January and 29 June 1998. The AOI data are observed as the daily market closing index value whereas the SPI data are observed as the last traded price on each day in the June 98 contract. Graphs of $\log$ AOI and $\log$ SPI in first differences are shown in figures 4 and 5. To test for the unitroots for each plotted series, we use Microfit 4.0 to carry out the augmented Dickey-Fuller (ADF) unit root test. ${ }^{3}$ The 95 percent critical values for each test computed using the response surface estimates indicate that both $\log$ AOI and log SPI are non-stationary but both $\Delta \log$ AOI and $\Delta \log$ SPI are stationary for the period 27 January 1998 to 17 June 1998 ( $\mathrm{T}=98) . \Delta \log$ AOI and $\Delta \log$ SPI continue to be stationary for the extended period from 27 January 1998 to 29 June ( $\mathrm{T}=106)$, where $\mathrm{T}$ is the sample size now under consideration.

The extended two-layered neural network described in section 2 is used to model the relationships between the AOI and SPI. In detecting the causal relationship from log SPI to

\footnotetext{
3 For simplicity, and to keep the paper to a reasonable size, the relevant ADF results are not presented, but can be supplied to readers upon request.
} 
$\log$ AOI, the variables used are $z(t)=\log$ AOI and $y(t)=\log$ SPI. As discussed above, neither $\log$ AOI nor log SPI are stationary. Therefore, we incorporate the forgetting factor, $\lambda$ to allow for the presence of non-stationarity. To begin, we first assume $\mathrm{Q}=16$, and then make use of the order update and the lattice-ladder recursions described above to select the 'optimal' specification of the distributed lag models at $\mathrm{T}=98,99, \ldots, 106$.

For the causal relationship from $\log$ SPI to $\log$ AOI, the optimal distributed lag models with $\lambda=0.99$ and 0.985 are presented in Table 2 . For brevity, only the results obtained by the MHQC are presented. For cases where $\lambda$ is less than 0.985 , the selected models are not reported due to the small effective sample size $(<50)$. The causal relationship from $\Delta \log$ SPI to $\Delta \log$ AOI was also examined and a model specification with lags 0,1 and 10 was selected by the MHQC at all times with $\lambda=1$. The lags 0,1 and 10 indicate the existence of instantaneous causality between $\Delta \log$ SPI to $\Delta \log$ AOI and one-day and two-week effects from $\Delta \log$ SPI to $\Delta \log$ AOI respectively.

To assess the causality from $\log$ SPI to $\log$ AOI, a subset model for $\lambda=0.99$ with lags $(0,1,10)$ was selected by the MHQC at $\mathrm{T}=98,99, \ldots, 102$. A two-layered neural network can be constructed for this model. At $\mathrm{T}=103$, the lag structure selected changes to $(0,10)$. The connection strength from the input unit representing $y(t-1)$ to the output unit has switched from non-zero to zero. In this case, the predicted output, log AOI, is fed by the current and previous inputs, log SPI. Also, the lag 0 indicates instantaneous causality between $\log$ AOI and $\log$ SPI. These results indicate that both instantaneous and direct causal relationships exist from the futures market to the stock market even when emphasis is placed more on the recent data. 
Figure 4. Log AOI in first differences, daily: 27 January 1998 to 29 June 1998

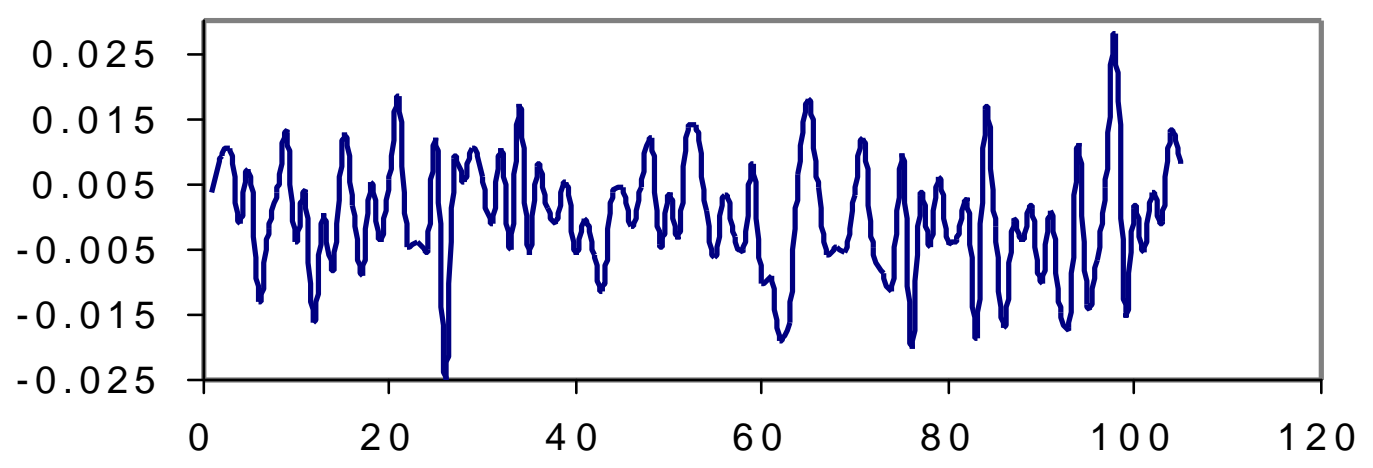

Figure 5. Log SPI in first differences, daily: 27 January 1998 to 29 June 1998

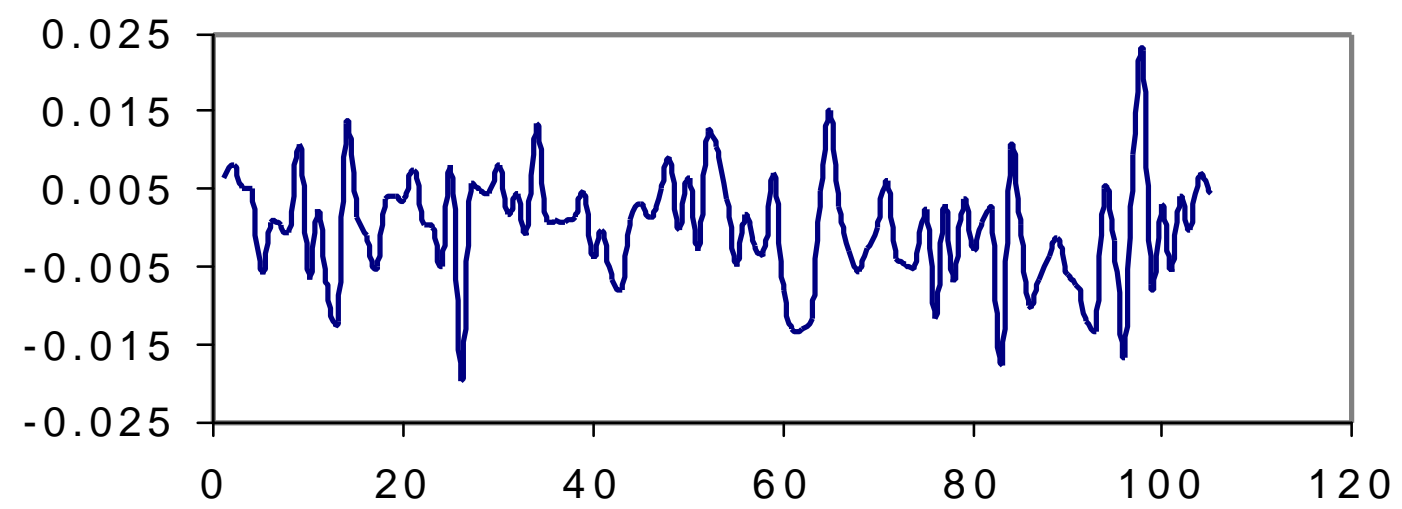

Figure 6. Log (Telstra's share price), daily: 24 November 1997 to 30 March 1998

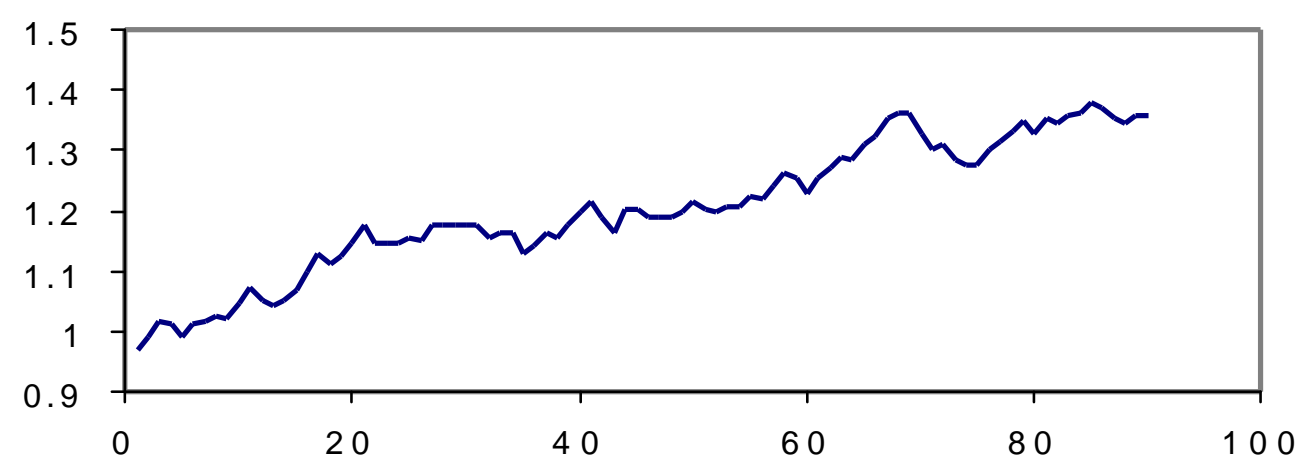


TABLE 2

The VRDLs selected by MHQC for detecting the causal relationship from SPI to AOI.

\begin{tabular}{|c|c|c|c|}
\hline \hline \multirow{2}{*}{$\begin{array}{c}\text { Sample size } \\
(\mathrm{T})\end{array}$} & \multicolumn{2}{|c|}{$\begin{array}{c}\text { Non-zero lag structure } \\
\text { for } \mathrm{z}(\mathrm{t})=\log \text { AOI and } \mathrm{y}(\mathrm{t})=\log \mathrm{SPI}\end{array}$} & \multirow{2}{*}{ Pattern of causality } \\
\cline { 2 - 3 } & $\lambda=0.99$ & $\lambda=0.985$ & \\
\hline $98,99,100,101,102$ & 0110 & 010 & $\log \mathrm{SPI} \rightarrow \log$ AOI \\
\hline $103,104,105,106$ & 010 & 010 & $\log \mathrm{SPI} \rightarrow \log$ AOI \\
\hline
\end{tabular}

(a) $\mathrm{y} \rightarrow \mathrm{z}$ : $\mathrm{y}$ causes $\mathrm{z}$ directly and instantaneously.

For the causal relationship from log AOI to log SPI, Table 3 shows the optimal distributed lag models with $\lambda=0.99$ and 0.985 . For the causal relationship from $\Delta \log$ AOI to $\Delta \log$ SPI, a model specification with lags 0,7 and 8 was selected by the MHQC at all times. These results strongly support the existence of instantaneous causal and bidirectional feedback relationships between the Australian stock and futures markets. These conclusions are generally consistent with those reported by Chan et al (1991) and Chan (1992).

TABLE 3

The VRDLs selected by MHQC for detecting the causal relationship from AOI to SPI.

\begin{tabular}{|c|c|c|c|}
\hline \hline \multirow{2}{*}{$\begin{array}{c}\text { Sample size } \\
(\mathrm{T})\end{array}$} & \multicolumn{2}{|c|}{$\begin{array}{c}\text { Non-zero Lag Structure } \\
\text { for } \mathrm{z}(\mathrm{t})=\log \text { SPI and } \mathrm{y}(\mathrm{t})=\log \text { AOI }\end{array}$} & \multirow{2}{*}{ Pattern of causality } \\
\cline { 2 - 3 } & $\lambda=0.99$ & $\lambda=0.985$ & \\
\hline $98,99,100,101,102$ & 0110 & 0110 & $\log \mathrm{AOI} \rightarrow \log \mathrm{SPI}$ \\
\hline $103,104,105,106$ & 010 & 010 & $\log \mathrm{AOI} \rightarrow \log \mathrm{SPI}$ \\
\hline
\end{tabular}

(a) $\mathrm{y} \rightrightarrows \mathrm{z}$ : $\mathrm{y}$ causes $\mathrm{z}$ directly and instantaneously.

There are other financial variables which could play a significant role in the stock-futures market analysis (see Brailsford and Hodgson (1997)). This application merely demonstrates 
the usefulness of the extended, two-layered, neural network structure and the learning algorithm in time series analysis, especially in a potentially non-stationary situation.

The second application examines the non-linearities in the relationship between share prices and the underlying stock market. We focus on the share price for Australia's Telstra Corporation Limited and the All Ordinaries Index. Telstra is one of Australia's largest companies. The traded shares represent the partially privatised Commonwealth Government's telecommunications organisation. The data used are daily series over the period 24 November 1997 to 30 March 1998 and were obtained from DataStream ${ }^{\mathrm{TM}}$. Graphs of log of Telstra's share price in levels is shown in figure 6. The 95 percent critical values for the augmented Dickey-Fuller (ADF) unit root test indicate that log of Telstra's share price is non-stationary. To demonstrate the usefulness of the proposed algorithm in a small sample environment, we carried out forecasting for period $(\mathrm{T}+1)$ by building subset VAR systems on the first T periods, using three-layer neural networks. We first detrend and mean-correct, rather than difference, the logarithms of the data for illustration purposes. Exponential forgetting was used with a forgetting factor 0.99 . The optimal subset VAR models with zero constraints for $T=75$ through $T=87$ were identified, where $y_{1}(t)=\log$ of Telstra's share price, $\mathrm{y}_{2}(\mathrm{t})=\log (\mathrm{AOI}), \mathrm{y}_{3}(\mathrm{t})=\mathrm{y}_{1}(\mathrm{t}) \mathrm{y}_{2}(\mathrm{t}), \mathrm{y}_{4}(\mathrm{t})=\mathrm{y}_{1}^{2}(\mathrm{t})$ and $\mathrm{y}_{5}(\mathrm{t})=\mathrm{y}_{2}^{2}(\mathrm{t})$. The specifications of all identified optimal VARs as a basis for constructing three-layer neural network are outlined in Table A.1 of the Appendix. One-step ahead forecasts based on each optimal VAR are calculated. For brevity, the forecasts are summarised in Table 4 . The forecasts based on the naive random-walk model are also shown for comparison purposes.

The Telstra share prices show a reasonable degree of volatility. Thus models which can correctly forecast whether the share price will rise, fall or stay constant are potentially quite useful for investors and financial market practitioners. In this regard, the optimal VARs 
appear to perform well. Of the fifteen signs (e.g. ten '+'s and five '-'s) of daily price change over the test period, VAR successfully predicts fourteen, whereas the random-walk model correctly forecasts only one. Theil's (1966) inequality coefficient, $\sqrt{\frac{\sum\left(\mathrm{P}_{\mathrm{i}}-\mathrm{A}_{\mathrm{i}}\right)^{2}}{\sum \mathrm{A}_{\mathrm{i}}^{2}}}$, is 0.0496 for the VAR forecast, where $\left(\mathrm{P}_{\mathrm{i}}, \mathrm{A}_{\mathrm{i}}\right)$ stands for a pair of predicted and observed changes. The RMS prediction error of the VAR forecast is $4.96 \%$ of the prediction error of the random-walk model forecast.

TABLE 4 VAR and Random-Walk forecasts: Telstra share price

\begin{tabular}{|c|c|c|c|c|c|c|c|c|}
\hline \multirow{3}{*}{$\begin{array}{c}\text { Trading days } \\
\text { in } \\
1998\end{array}$} & \multirow{3}{*}{$\mathrm{T}$} & \multicolumn{2}{|c|}{$\begin{array}{c}\text { Actual figures } \\
\text { Telstra }\end{array}$} & \multicolumn{3}{|c|}{ VAR } & \multicolumn{2}{|c|}{ Random Walk } \\
\hline & & \multirow{2}{*}{ SharePrice } & \multirow{2}{*}{$\begin{array}{l}\text { Daily Price } \\
\text { Change }\end{array}$} & \multicolumn{2}{|c|}{ Forecast } & \multirow{2}{*}{$\begin{array}{l}\text { Change } \\
\text { Predicted }\end{array}$} & \multicolumn{2}{|c|}{ Forecast } \\
\hline & & & & Value & Error & & Value & Error \\
\hline 10 March & 77 & 3.67 & +0.09 & 3.64 & -0.03 & +0.06 & 3.58 & -0.09 \\
\hline 11 March & 78 & 3.73 & +0.06 & 3.73 & +0.00 & +0.06 & 3.67 & -0.06 \\
\hline 12 March & 79 & 3.79 & +0.06 & 3.76 & -0.03 & +0.03 & 3.73 & -0.06 \\
\hline $13 \mathrm{March}$ & 80 & 3.85 & +0.06 & 3.82 & -0.03 & +0.03 & 3.79 & -0.06 \\
\hline 16 March & 81 & 3.78 & -0.07 & 3.82 & 0.04 & -0.03 & 3.85 & 0.07 \\
\hline 17 March & 82 & 3.87 & +0.09 & 3.86 & -0.01 & +0.08 & 3.78 & -0.09 \\
\hline $18 \mathrm{March}$ & 83 & 3.84 & -0.03 & 3.83 & -0.01 & -0.04 & 3.87 & 0.03 \\
\hline 19 March & 84 & 3.89 & +0.05 & 3.86 & -0.03 & +0.02 & 3.84 & -0.05 \\
\hline $20 \mathrm{March}$ & 85 & 3.91 & +0.02 & 3.90 & -0.01 & +0.01 & 3.89 & -0.02 \\
\hline $23 \mathrm{March}$ & 86 & 3.98 & +0.07 & 3.94 & -0.04 & +0.03 & 3.91 & -0.07 \\
\hline 24 March & 87 & 3.94 & -0.04 & 3.96 & 0.02 & -0.02 & 3.98 & 0.04 \\
\hline $25 \mathrm{March}$ & 88 & 3.87 & -0.07 & 3.93 & 0.06 & -0.01 & 3.94 & 0.07 \\
\hline 26 March & 89 & 3.84 & -0.03 & 3.87 & 0.03 & +0.00 & 3.87 & 0.03 \\
\hline 27 March & 90 & 3.89 & +0.05 & 3.87 & -0.02 & +0.03 & 3.84 & -0.05 \\
\hline $30 \mathrm{March}$ & 91 & 3.89 & +0.00 & 3.89 & +0.00 & +0.00 & 3.89 & +0.00 \\
\hline \multicolumn{3}{|l|}{ Average } & 0.021 & & -0.004 & 0.017 & & -0.021 \\
\hline
\end{tabular}

Of course, we are aware that many other non-parametric regression techniques and dynamic forgetting factor methods could play a significant role in forecasting. Our interest in this study is mainly to investigate how the algorithm proposed in section 3 may be utilised to predict the direction of change using a volatile financial market as an example. Possible algorithms to improve the accuracy of the forecast magnitude are currently under investigation. 


\section{CONCLUSION}

In this paper, a numerically robust lattice-ladder learning algorithm has been developed to sequentially select the best specification for a subset time series system using neural networks. An extended two-layered neural net is developed to model the VRDL system with a subset structure. As the neural input vector, $y(t)$, includes the second-order terms, the neural net, with slight modification, is extended to the cases of three-layers. The proposed construction method is simple to use and can be applied to an M-layered polynomial neural network with hidden layer nodes in layer $m \in[1, \mathrm{M}-2]$. The overall lattice-ladder learning algorithm for extended neural networks avoids cumbersome matrix inversion and enjoys better numerical accuracy. Two applications have been used to illustrate the practicality of the process. However, further study will be planned to extend Hall's (1990) bootstrap approach to evaluate the performance reliability of extended neural networks. 


\section{REFERENCES}

Brailsford, T.J. and Hodgson, A. (1997) Mispricing in Stock Index Futures: A Re-examination Using the SPI, Australian Journal of Management, 22, 21-43.

Bollerslev, T., Chou, R.Y. and Kroner, K.F. (1992) ARCH Modeling in Finance: A Review of the Theory and Empirical Evidence, Journal of Econometrics, 52, 5-29.

Carayannis, C., Manolakis, C.D. and Kalouptsidis, N. (1986) A Unified View of Parametric Processing Algorithms for Pre-windowed Signals, Signal Processing, 10, 335-368.

Chakraborty, K., Mehrotra, K., Mohan, C. and Ranka, S. (1992) Forecasting the Behavior of Multivariate Time Series Using Neural Networks, Neural Networks, 5, 961-970.

Chan, K. (1992) A Further Analysis of the Lead-Lag Relationship Between the Cash and Stock Index Futures Market, Review of Financial Studies, 5, 123-151.

Chan, K., Chan, K.C. and Karolyi, G.A. (1991) Intraday Volatility in the Stock Index and Stock Index Futures Markets, Review of Financial Studies, 4, 657-684.

Chung, Y.P. (1991) A Transactions Data Test of Stock Index Futures Market Efficiency and Index Arbitrage Profitability, Journal of Finance, 46, 1791-1809.

Farlow,S.J.(1984) Ed., Self-Organizing Methods in Modeling: GMDH Type Algorithms, vol. 54 of Statistics: Textbooks and Monographs. New York: Marcel Dekker.

Geweke, J.F. and Singleton, K.J. (1981) Maximum likelihood 'Confirmatory' Factor Analysis of Economic Time series, International Economic Review, 22, 37-54

Hall, P. (1990) Using the Bootstrap to Estimate Mean Squared Error and Select Smoothing Parameter in Nonparametric Problems, Journal of Multivariate Analysis, 32, 177-203.

Hannan, E.J. and Deistler, M. (1988) The Statistical Theory of Linear Systems, John Wiley \& Sons, Inc. New York.

Holmes, J.M. and Hutton, P.A. (1989) 'Optimal' Model Selection When the True Relationship is Weak and Occurs with a Delay, Economics Letters, 30, 333-339.

Kwok, T.Y. and Yeung, D.Y. (1997) Constructive Algorithms for structure Learning in Feedforward Neural Networks for Regression Problems, IEEE Trans. on Neural Networks, 8, May, 630-645.

MacKinlay, A. and Ramaswamy, K. (1988) Index-Futures Arbitrage and the Behaviour of Stock Index Futures Prices, Review of Financial Studies, 1, 137-158.

Martens, M., Kofman, P. and Vorst, T. (1996) A Threshold Error Correction Model for Intraday Futures and Index Returns, Working paper, Erasmus University Rotterdam.

Mittnik,S (1989) Forecasting With Balanced State Space Representations of Multivariate Distributed Lag Models, Journal of Forecasting, 9, 207-218. 
Penm, J.H.W. and Terrell, R.D. (1982) On the Recursive Fitting of Subset Autoregressions, Journal of Time Series Analysis, 3, 43-59.

Penm, J.H.W. and Terrell, R.D. (1984) Multivariate Subset Autoregressive Modelling with Zero Constraints for Detecting Causality, Journal of Econometrics, Feb. 311-330.

Penm, J.H.W., Penm, J.H. and Terrell, R.D. (1992) Using the Bootstrap as an Aid in Choosing the Approximate Representation for Vector Time Series, Journal of Business and Economic Statistics, 10, 213-219.

Penm, J.H.W., Penm, J.H. and Terrell, R.D. (1995) A Note on the sequential Fitting of Multichannel Subset Autoregressions Using the Prewindowed Case, IEEE Trans. on Signal Processing, 43, 322-327.

Petri, C. (1979), Introduction to General Net Theory, Lecture Notes in Computer Science, Berlin: Springer-Verlag.

Refenes, A., Abu-Mustafa, Y., Moody, J. and Weigend, A. (1996) eds, Neural Networks in Financial Engineering, vol. 2 of Progress in Neural Processing, World Scientific Publishing, Co., London.

Teräsvirta, T., Lin, C. and Granger, C.W.J. (1993) Power of the Neural Network Linearity Test, Journal of Time Series Analysis, 14, 209-220.

Theil, H. (1966), Ed., Applied Economic Forecasting, North-Holland Publishing Company, Amsterdam.

Tse, Y.K. (1995) Lead-Lag Relationship Between Spot Index and Futures Prices of the Nikkei Stock Average, Journal of Forecasting, 14, 553-563.

Watanabe, K.C., Fukuda, T. and Tzafestas, S.G. (1992) An Adaptive Control for CARMA Systems Using linear Neural Networks, Int. J.Control, 56, 483-497. 
Appendix: In this appendix, the identified optimal VARs for forecasting the Telstra share prices as a basis for constructing three-layer neural networks are outlined in Table A.1

TABLE A.1 The optimal subset VAR models selected for MHQC

Variables: $\mathrm{y}_{1}(\mathrm{t})=\log \left(\right.$ Telstra's share price), $\mathrm{y}_{2}(\mathrm{t})=\log (\mathrm{AOI}), \mathrm{y}_{3}(\mathrm{t})=\mathrm{y}_{1}(\mathrm{t}) \mathrm{y}_{2}(\mathrm{t}), \mathrm{y}_{4}(\mathrm{t})=\mathrm{y}_{1}{ }^{2}(\mathrm{t})$ and $\mathrm{y}_{5}(\mathrm{t})=\mathrm{y}_{2}{ }^{2}(\mathrm{t})$. Estimated standard errors in parentheses

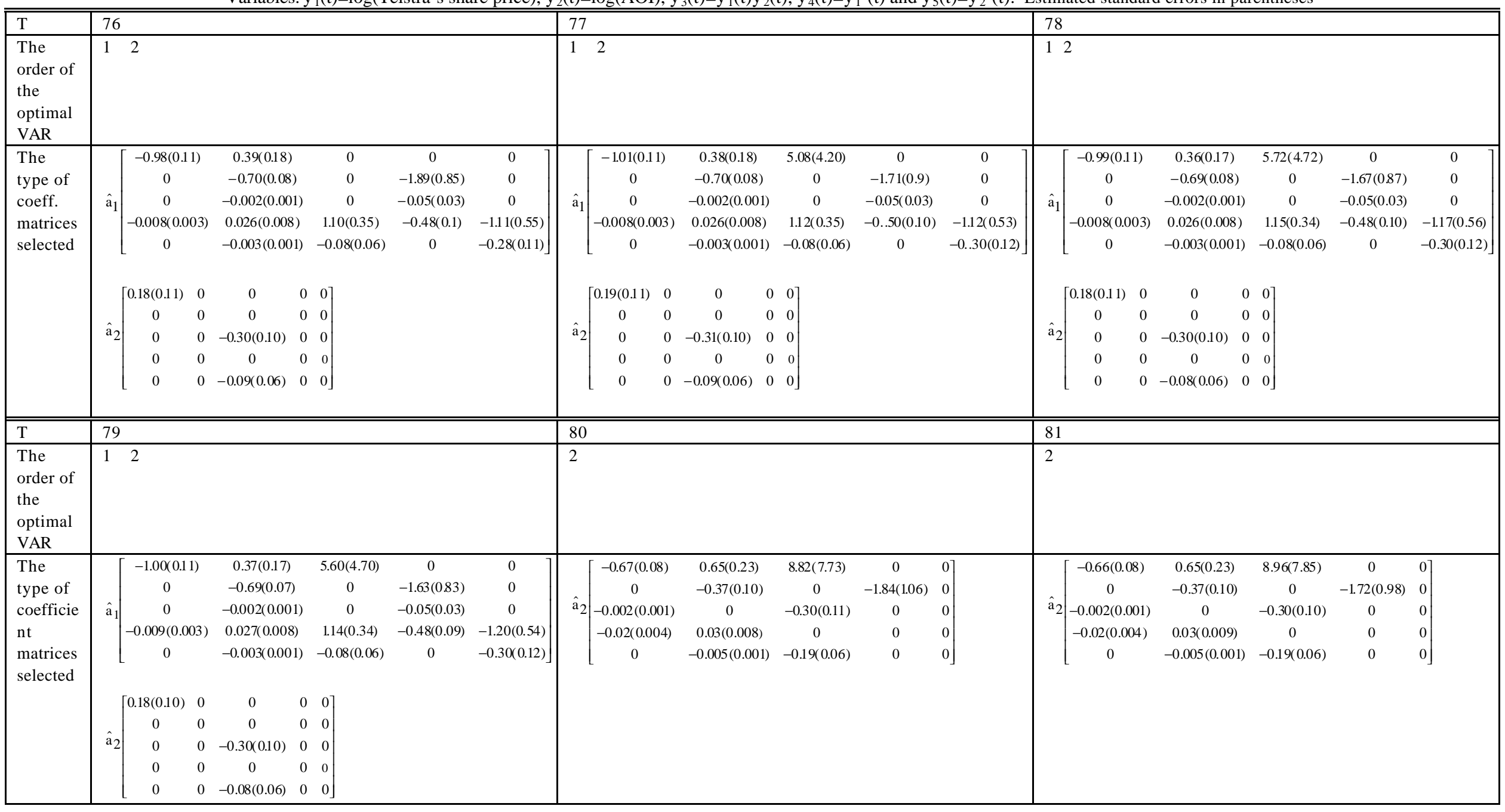




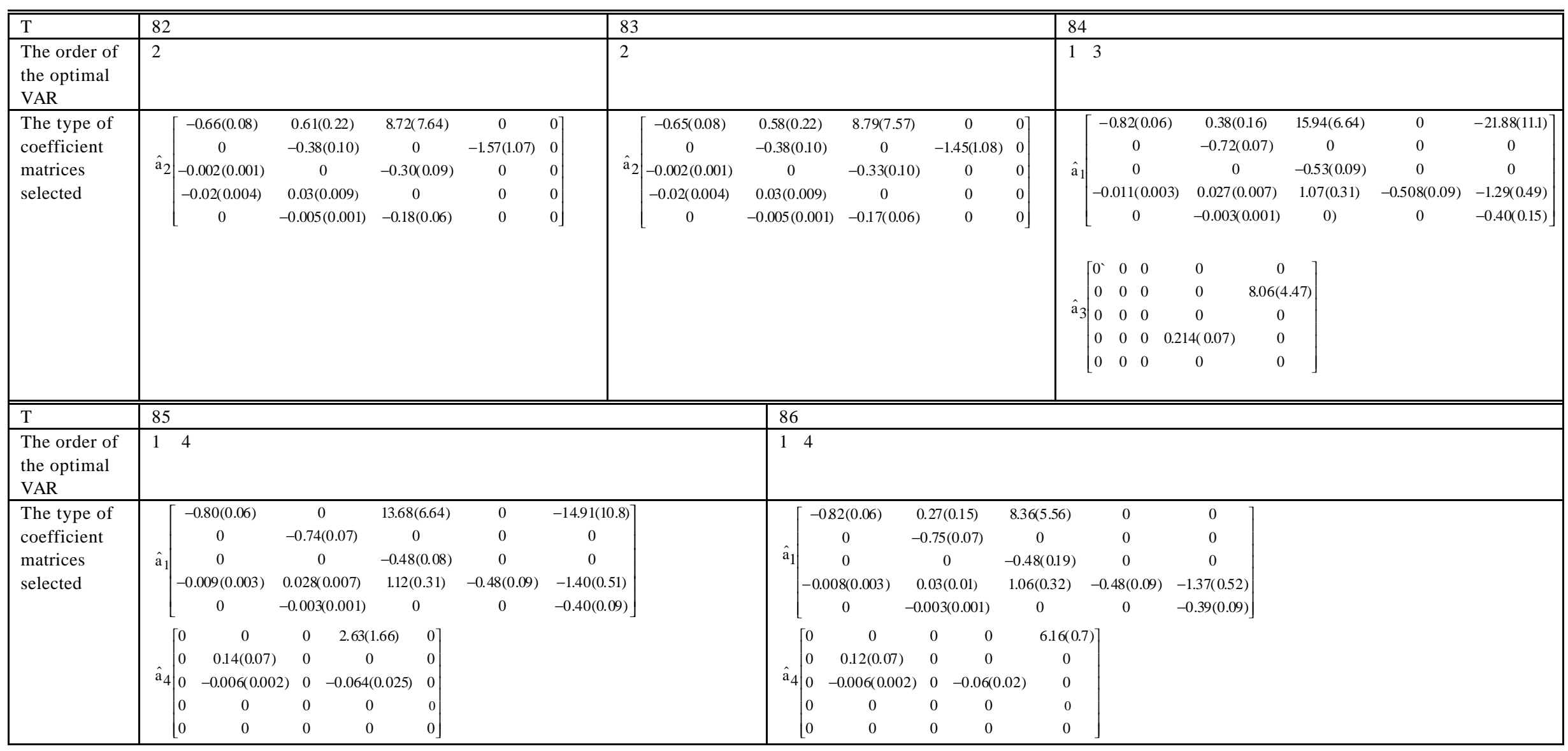




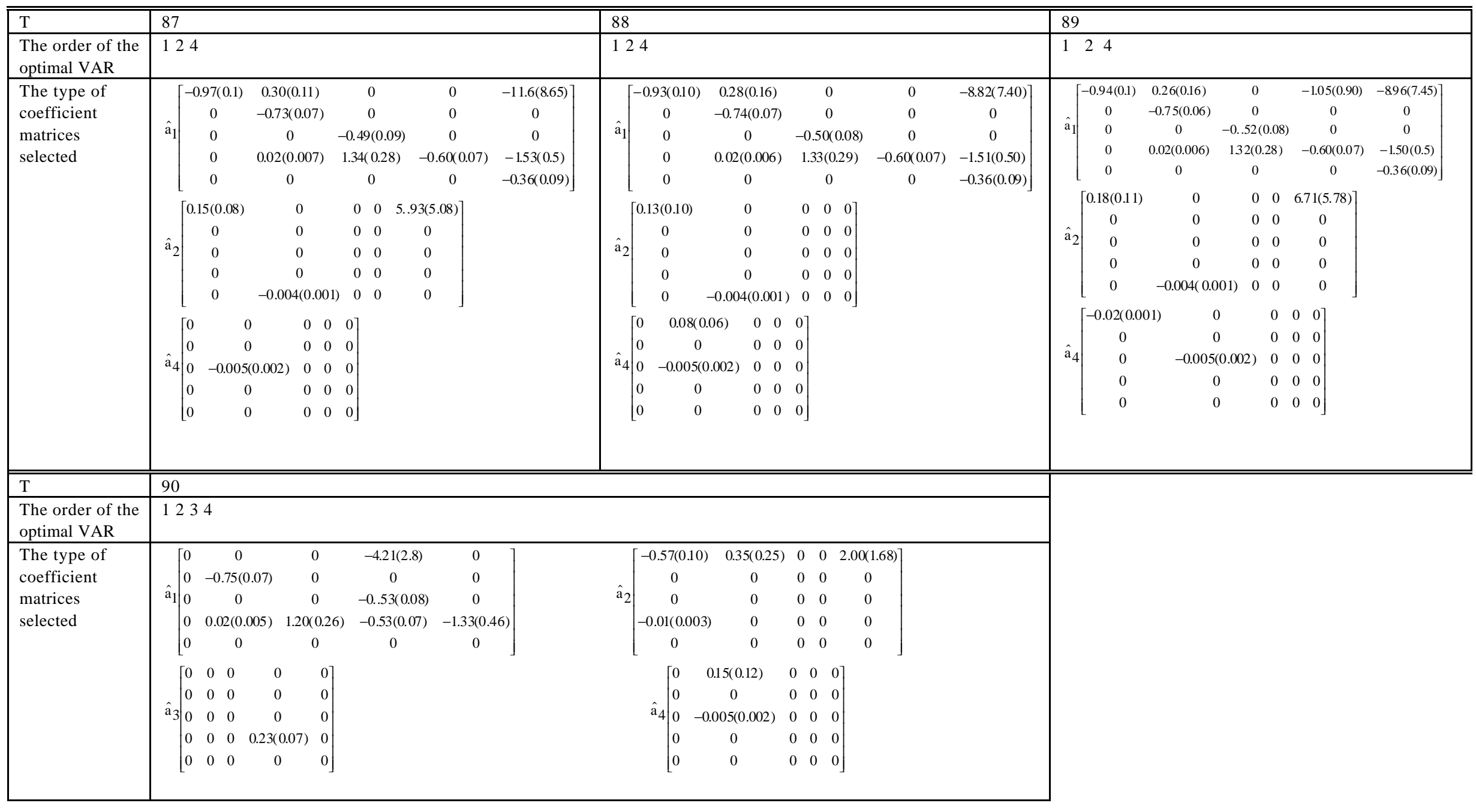

